\title{
COMBINATION OF MOBILE AGENT AND EVOLUTIONARY ALGORITHM TO OPTIMIZE THE CLIENT TRANSPORT SERVICES
}

\author{
Hayfa ZgayA $^{1}$, Slim Hammadi ${ }^{1}$ AND Khaled GhÉdira ${ }^{2}$
}

\begin{abstract}
This paper presents a migration strategy for a set of mobile agents (MAs) in order to satisfy customers' requests in a transport network, through a multimodal information system. In this context, we propose an optimization solution which operates on two levels. The first one aims to constitute a set of MAs building their routes, called Workplans. At this level, Workplans must incorporate all nodes, representing information providers in the multimodal network, in order to explore it completely. Thanks to an evolutionary approach, the second level must optimize nodes selection in order to increase the number of satisfied users. The assignment of network nodes to the required services must be followed by a Workplan update procedure in order to deduce final routes paths. Finally, simulation results are mentioned to invoke the different steps of our adopted approach.
\end{abstract}

Keywords. Mobile agents, evolutionary algorithms, multimodal information system, multimodal transport network.

Mathematics Subject Classification. 90B06, 90C29, 68M14, $68 \mathrm{~T} 20,68 \mathrm{U} 35$.

\section{INTRODUCTION}

According to the exponential growth of the services and information available on large networks such as the Internet, multimodal transport network customers

Received 30 October, 2006. Accepted 12 October, 2007.

1 LAGIS UMR 8146 École Centrale de Lille, France;

hayfa.zgaya@ec-lille.fr; slim.hammadi@ec-lille.fr

2 SOIE, ISG-Tunis ISG, Tunis University, Tunisia; khaled.ghedira@isg.rnu.tn 
require relevant, interactive and instantaneous information during their travels. A Multimodal Information System (MIS) can offer support tool in order to make available needed information. The main goal of such a system is to guide and help network customers to make good decisions when they are travelling. Information is represented by distributed data located on different nodes through a Multimodal Network (MN). These nodes represent servers which are owners of multimodal transport, corresponding to our system information providers. In a previous work [1], we proposed a MIS based on a special kind of software agent called Mobile Agent (MA). A MA [2] is a program composed of code, data and a state, able to move from a node to another through a network to perform tasks and finally return to its original node called Home [4-6]. MA technology can considerably reduce network traffic [3] as such is the case in the field of the data extraction which can require the access to huge and geographically separated data sources [7]. In fact, instead of transferring huge amount of data through the network, a MA migrates to concerned information servers and performs its code using the resources of the visited nodes to collect needed data. Finally, this agent returns to the Home node with expected results. In this paper, we present a migration strategy of MAs in order to achieve user satisfaction in term of cost and response delay. Therefore, we propose an optimization solution which operates on two levels. The first one aims to optimize the number of MAs in order to explore the whole MN respecting initial routes, which are provisional. Final routes are deduced every time a set of customers' simultaneous requests is formulated. In this case, the MIS identifies the set of nodes which propose required services. From this set and thanks to an evolutionary method, the system optimizes the selection of nodes to answer to formulated requests; this last procedure represents the second level of our solution. Selected nodes are chosen according to the total resulted cost and response delay corresponding to generated responses. A customer is satisfied if he obtains rapidly a response to his request, with a suitable cost. This paper is organized in seven parts. In the following section, we present the problem. Then, we detail our proposed solution within two steps through Sections 4 and 5 which represent respectively the first and second level of the adopted strategy. Simulation results are presented in Section 6 and the paper is concluded in last section.

\section{PROBLEM DESCRIPTION}

New distributed applications are spread on huge networks, employing heterogeneous and voluminous data sources located on geographically separated sites. Through such networks, different actions (navigation, requests, storage, update, etc) can be launched intensely and in a simultaneous way requiring the access to several heterogeneous and distant data sources. This behaviour can be illustrated by different kinds of interactions among individual entities [8]. Thus, a multi-agent approach seems to be the most appropriate to implement the complex character of this kind of system. Besides, distributed applications through wide networks are not easy to realize because of the limited aspect of bandwidth which remains 


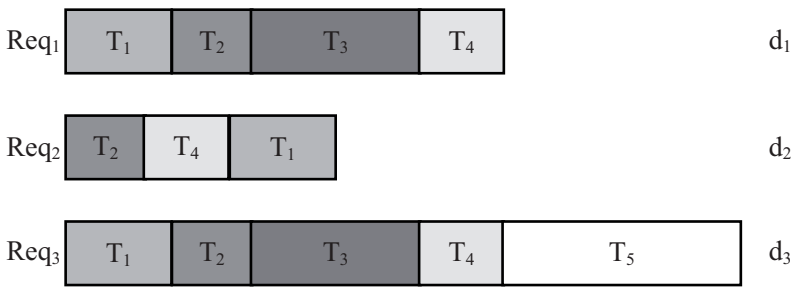

Figure 1. Requests decomposition.

restricted by several technical factors [9]. Therefore, permanent connexion can not be constantly available. That's why, we have to manage data information availability despite recurrent connexions in order to access and share distributed data. In this context, mobile technology [10] can complement artificial intelligence because it can reduce considerably network traffic [3]. Giving the mobility character to a software agent will allow it to migrate towards any node on the network which can receive mobile entities. Our goal is to design and implement a MIS which is able to receive and send mobile entities through huge and distributed networks, in order to optimize the management of the responses to intensive and simultaneous requests. We aim to extend our approach to new distributed applications (PDA, laptop ...) corresponding to wireless technology generation where bandwidth remains limited. In this paper, we are interested to satisfy MN customers; this is a two-steps assignment problem: firstly the assignment of network nodes to MAs to build their routes, called Workplans and then, a sub-set of these nodes are selected to assign tasks, in order to satisfy users. A task is an independent sub-request which belongs to one or several requests formulated simultaneously (Fig. 1). After that, information providers which propose services corresponding to identify tasks are recognized (Fig. 2). Finally, nodes must be assigned to tasks in order to satisfy all connected users (Fig. 3). A user is satisfied if his request was answered rapidly with a reasonable cost.

The problem is defined by:

- $R$ requests, waiting for responses at the same instant $t$. The set of these requests is noted $R_{t}$,

- Each request $r e q_{w} \in R_{t}$ is decomposed into a set of independent tasks, noted $I_{t, w}$,

- Each request $r e q_{w}$ has a due date $d_{w}$ initially known (Fig. 1), an ending date $D_{w}$ and a total cost $C_{w}$,

- The set of independent $I$ tasks representing all proposed services on the $\mathrm{MN}$ is noted $I_{t}$,

- The realization of each task $T_{i} \in I_{t}$ requires a resource, or node, selected from a set of J available nodes, noted $S=\left\{S_{1}, \ldots, S_{J}\right\}$,

- The set of independent $I^{\prime} \operatorname{tasks}\left(I^{\prime} \leqslant I\right)$ composing $R_{t}$ is noted $I_{t}^{\prime}\left(I_{t}^{\prime} \subseteq\right.$ $\left.I_{t}\right)$ 

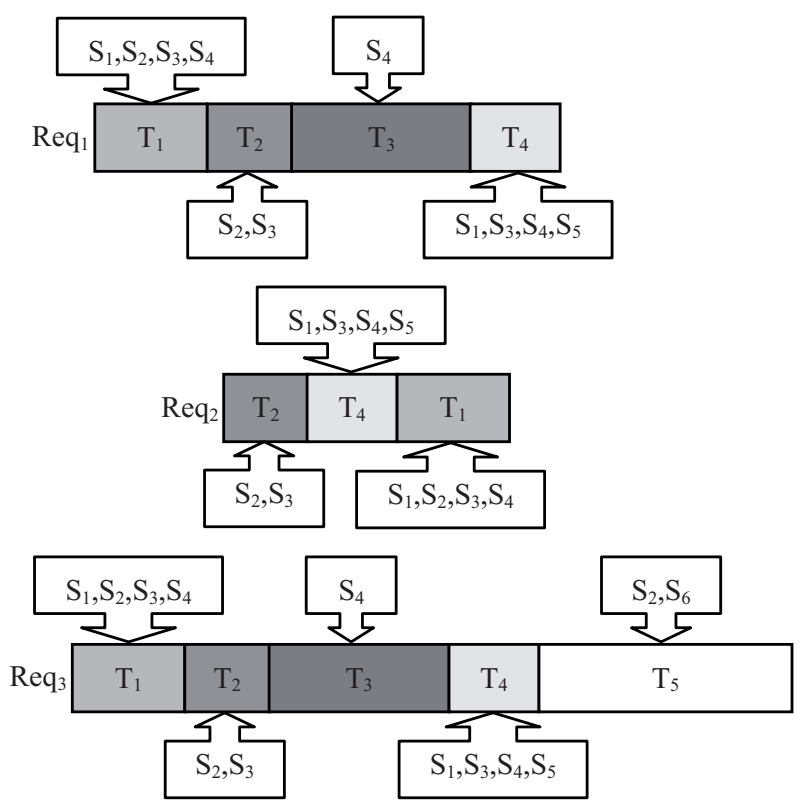

Figure 2. Nodes identification.
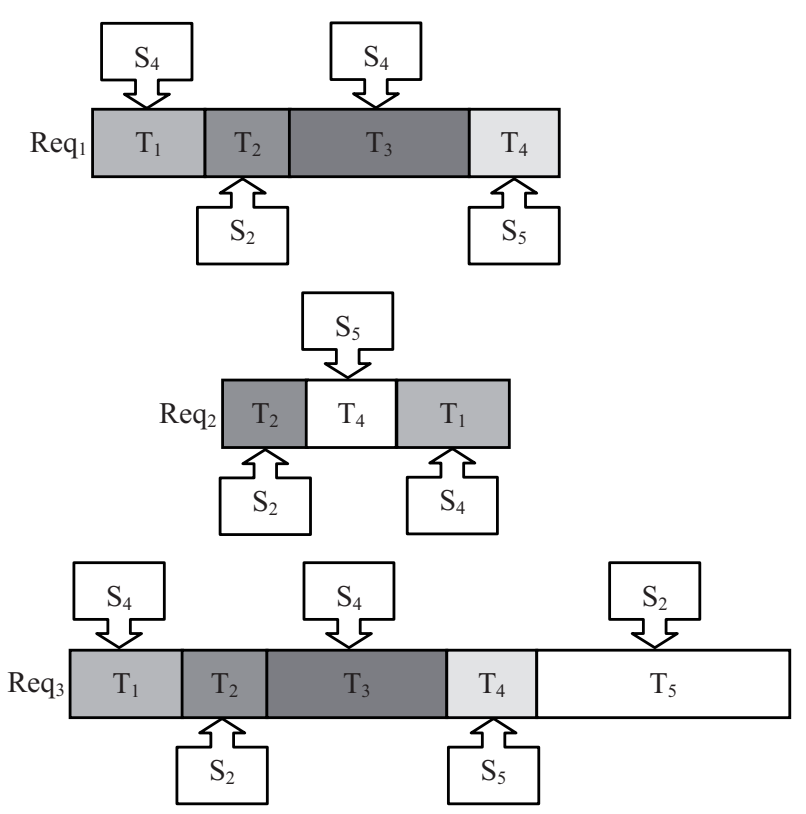

Figure 3. Nodes assignment. 
TABle 1. Example of available services (Processing time, cost and data size).

\begin{tabular}{|l|l|l|l|l|}
\hline & $S_{1}$ & $S_{2}$ & $S_{3}$ & $S_{4}$ \\
\hline$T_{1}$ & $(0,0,0)$ & $(2,5,3)$ & $(4,3,3)$ & $(2,5,3)$ \\
\hline$T_{2}$ & $(2,4,5)$ & $(1,5,2)$ & $(4,5,1)$ & $(3,8,3)$ \\
\hline$T_{3}$ & $(1,7,3)$ & $(0,0,0)$ & $(2,5,3)$ & $(4,2,2)$ \\
\hline$T_{4}$ & $(3,2,1)$ & $(0,0,0)$ & $(0,0,0)$ & $(0,0,0)$ \\
\hline$T_{5}$ & $(2,3,1)$ & $(1,1,3)$ & $(4,5,2)$ & $(4,5,3)$ \\
\hline
\end{tabular}

- The set of $J^{\prime}$ nodes $\left(J^{\prime} \leqslant J\right)$ selected from $S$, to perform $I_{t}^{\prime}$ is noted $S^{\prime}$ $\left(S^{\prime} \subseteq S\right)$,

- There is a predefined set of processing time; for a given node $S_{j}$ and a given task $T_{i}$, the processing time of $T_{i}$ using $S_{j}$ is defined and noted by $P_{i j}$

- There is a predefined set of information cost; for a given node $S_{j}$ and a given task $T_{i}$, the cost of the information to collect from $S_{j}$, corresponding to the service referenced by $T_{i}$, is defined and noted by $\mathrm{Co}_{i j}$,

- The size of the data to collect to ensure a service is defined; for a given node $S_{j}$ and a given task $T_{i}$, the data size is defined and noted by $Q_{i j}$,

- We have partial flexibility, the realisation of each task $T_{i}$ requires a node selected from a set of nodes which propose the same service performing the task $T_{i}$, with different cost, processing time and data size. Therefore, a service is described by:

(i) A processing time $P_{i j}$ of the task $T_{i}$ on the node $S_{j}$. It corresponds to the estimated time to perform the task $T_{i}$ by means of the resources of $S_{j}$

(ii) The cost of the service $C o_{i j}$ corresponding to the task $T_{i}$ on the node $S_{j}$,

(iii) The data size $Q_{i j}$ corresponding to the size of the information to collect from $S_{j}$ to response to $T_{i}$.

A same task may be performed differently on several nodes, namely with different processing time, different cost and different response formats. These three characteristics $\left(P_{i j}, C_{i j}, Q_{i j}\right)$ represent successively the first, second and last term of each element of a service table. Table 1 below shows an example of different proposed services. We notice that if a provider does not offer a response to a task (partial flexibility); the correspondent term in the table above is $(0,0,0)$. Otherwise we have $P_{i j} \neq 0, C o_{i j} \neq 0$ and $Q_{i j} \neq 0$.

An analogy between the problem described above and the Flexible Job Shop Problem (FJSP) can be illustrated as follows in Table 2:

Apart from the analogy expressed in Table 2 above, we manage in our problem the similarities of requests in order to avoid the same data research. Besides, we have to assign the tasks to servers (cost and delay criteria) as well as to assign remote nodes (servers) to MAs taking into account the network state (latency 
TABLE 2. Analogy between FJSP and our problem.

\begin{tabular}{|c|c|}
\hline FJSP & Our Problem \\
\hline$N$ jobs & $R$ requests \\
\hline$M$ machines & $\mathrm{J}$ servers \\
\hline $\begin{array}{l}\mathrm{n}_{\mathrm{j}} \text { non preemptable } \\
\text { ordered operations } / \text { job }_{\mathrm{j}}\end{array}$ & $\begin{array}{l}\mathrm{n}_{\mathrm{j}} \text { non preemptable } \\
\text { ordered tasks/request }{ }_{\mathrm{j}}\end{array}$ \\
\hline $\begin{array}{l}\text { The problem: to organize } \\
\text { the execution of } \mathrm{N} \text { jobs on } \\
\text { M machines }\end{array}$ & $\begin{array}{l}\text { The problem: to organize } \\
\text { the execution of } \mathrm{R} \\
\text { requests on } \mathrm{S} \text { servers }\end{array}$ \\
\hline $\begin{array}{l}\text { The execution of each } \\
\text { operation } \mathrm{i} \text { of a job } \mathrm{j}\left(\mathrm{O}_{\mathrm{i}, \mathrm{j}}\right) \\
\text { requires one resource or } \\
\text { machine selected from a } \\
\text { set of available machines }\end{array}$ & $\begin{array}{l}\text { The execution of each } \\
\text { task } \mathrm{i}\left(\mathrm{T}_{\mathrm{i}}\right) \text { of a request } \mathrm{j} \\
\left(\text { req }_{\mathrm{i}}\right) \text { requires one } \\
\text { resource or server } \\
\text { selected from a set of } \\
\text { available servers } \\
\text { (similarities of requests) }\end{array}$ \\
\hline $\begin{array}{l}\text { The assignment of the } \\
\text { operation } \mathrm{O}_{\mathrm{i}, \mathrm{j}} \text { to the } \\
\text { machine } \mathrm{M}_{\mathrm{k}} \text { entails the } \\
\text { occupation of this } \\
\text { machine during a } \\
\text { processing time called } \mathrm{d}_{i, j, k}\end{array}$ & $\begin{array}{l}\text { The assignment of the } \\
\text { task } T_{i} \text { to the server } S_{k} \\
\text { entails the occupation of } \\
\text { this server during a } \\
\text { processing time called } \mathrm{P}_{i k}\end{array}$ \\
\hline $\begin{array}{l}\text { At a given time, a machine } \\
\text { can only execute one } \\
\text { operation: it becomes } \\
\text { available to other } \\
\text { operations one the } \\
\text { operation which is } \\
\text { currently assigned to is } \\
\text { completed (resource } \\
\text { constraints). }\end{array}$ & $\begin{array}{l}\text { At a given time, a server } \\
\text { can only execute one } \\
\text { task: it becomes available } \\
\text { to other tasks one the } \\
\text { task which is currently } \\
\text { assigned to is completed } \\
\text { (resource constraints). }\end{array}$ \\
\hline
\end{tabular}

criterion). Therefore our problem presents more difficulty than the FJSP which has been shown to be NP-hard.

\section{Adopted APPROACH}

To resolve the problem described in last section, we propose a system based on the coordination of five kinds of software agents [8,21] (Fig. 4):

(1) Interface Agents (IA). These agents interact with system users, allowing them to choose appropriate form of responses to their demands so IA agents manage requests and then display results. When a MN customer access to the MIS, an agent IA deals with the formulation of his request 


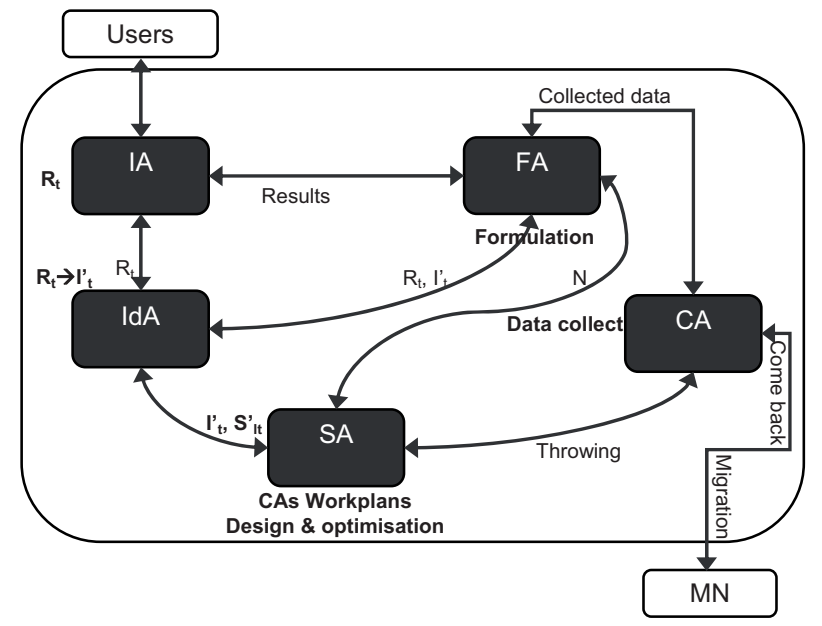

Figure 4. Multi-agent approach.

and then sends it to an available identifier agent. This one relates to the same platform to which several users can be simultaneously connected, thus it can receive several requests formulated at the same time. An identifier agent has to identify and to choose nodes which propose services corresponding to the requests of the users.

(2) Identifier agents (IdA). These agents decompose received requests into sub-requests corresponding, for example, to sub-routes or to well-known geographical zones. Sub-requests are elementary independent tasks to be performed by the available set of nodes (information providers) on the MN. Each node must login to the system registering all proposed services. A service corresponds to the response to a defined task with fixed cost, processing time and data size. Therefore, the agent IdA decomposes the set of simultaneous available requests into a set of independent tasks recognizing possible similarities, in order to avoid a redundant data research. The decomposition process occurs during the identification of the information providers. Finally, the agent IdA transmits all generated data to available scheduler agents which must optimize the choice of MN nodes, taking into account some system constraints.

(3) Scheduler Agents (SA). Several nodes may propose the same service with different cost and processing time and data size. The agent SA has to assign nodes to tasks minimizing total cost and processing time in order to respect due dates (data constraint). Selected set of nodes corresponds to the sequence of nodes building Workplans (routes) of the data collector agents. The agent SA has firstly to find an effective number of collector agents then he has to optimize the assignments of nodes to different tasks. This behaviour will be developed later. 
(4) Collector agents ( $C A$ ). An agent CA is a mobile software agent which can move from a node to another through a network in order to collect needed data. This special kind of agent is composed of data, code and a state [2]. Collected data should not exceed a capacity threshold in order to avoid overloading the MA. Therefore, the agent SA must take into account this aspect when assigning nodes to tasks. When they come back to the system, the agents AC must transmit collected data to available fusion agents.

(5) Fusion Agents (FA). These agents have to fusion correctly collected data in order to compose responses to simultaneous requests. The fusion procedure progresses according to the collected data availability. Each new answer component must be complementary to the already merged ones. Providers are already selected and tasks are supposed independent. Therefore, there is no possible conflict. A response to a request may be complete if a full answer is ready because all concerned components are available. It can be partial if at least a task composing the request was not treated, for example, because of an unavailable service. Finally, a response can be null if no component is available. If an answer is partial, the correspondent result is transmitted to the concerned user through the agent IA which deals with request reformulation, with or without the intervention of the user.

The agent IdA manages the simultaneous requests formulated by the users, decomposing them into a set of independent tasks. The decomposition process includes the identification of the requests similarities in order to formulate a set of autonomous and independent tasks which are waiting for responses at the same time $t$. Each task represents a service which can be proposed by different MN nodes, with different cost, processing time and data size. To response to tasks, needed data is available through the MN and their collect correspond to the CA agents job. Therefore, the SA agent must optimize the assignments of nodes to tasks, minimizing total cost and processing time, in order to respect due dates. To this assignment problem, we propose a two-level optimization solution. The first level aims to find a suitable number of CAs building their initial Workplans in order to explore the MN completely. The second level corresponds to the nodes selection increasing the number of satisfied users. These two complementary levels are detailed respectively through Sections 4 and 5 .

\section{The WORKPLAN PROBLEM}

Latency is the needed time for a data packet to cross a network connection, from sender to receiver. It varies according network state and can affect data transmission through distributed applications. Therefore, using CA agents can decrease considerably network traffic because they do not require simultaneous connection among different nodes [3]. In this section, we consider the Workplans design problem [11, 12, 13]. In a previous work [14], we proposed a Workplan design scheme which aims to find a suitable number of CA agents minimizing 
their navigation time in order to explore all MN nodes, taking into account network latency.

\section{A. Hypothesis}

In this part, we consider the following assumptions:

- Collecting data on a visited node uses processing time. We suppose that the size of the data to collect from a network node is equal to the average of the total data size on this node,

- Initially, we assume that a CA agent is not totally empty because it contains an initial quantity of data $\mathrm{Q}_{0}$,

- We suppose that minimal latency between each pair of nodes of the network is available thanks to an existant network monitoring module,

- Information can have a multimedia aspect, so we can assume that the transmission of a quantity of data from a node to another depends on current latency.

\section{B. DESCRIPTION}

The MA Workplan problem can be described as follows:

$\mathrm{AC}$ agents are created and initially launched from an originally node (Home node). The other network nodes represent available information providers where a CA agent can move to collect data corresponding to claimed services. These ones are expressed in term of independent tasks. A same service can be proposed by different nodes with different cost, processing time and different formats. On Home node, processing time is null. We call a service response time on a node, the processing time of the correspondent task to this service on this node. Latencies are known and may affect navigation time of CA agents. Our goal is to minimize CA agent number and their navigation time in order to explore all the $\mathrm{MN}$, taking into account network latency. We introduce some definitions using variables described in Table 3 below.

Definition 1. $L_{\min }\left(S_{i}, S_{j}\right)$ (Shortest latency between nodes $S_{i}$ and $S_{j}$ ) evaluated by shortest paths linking all pairs of nodes [15]. It corresponds to the minimum length of time needed to transfer a data packet from $S_{i}$ to $S_{j}$.

Definition 2. $C T_{i}$ (Processing time on node $S_{i}$ ) corresponds to needed computing time on the node $S_{i}$ to extract the data quantity $Q t_{i}$.

Definition 3. $Q t_{i}$ (Data quantity on node $S_{i}$ ) it is the average data size to extract from $S_{i}$.

$T_{i}$ represents a task corresponding to a service proposed by $S_{j}$. Therefore if $n t_{j}$ is the number of all available services on the node $S_{j}$ then (Sect. 2):

$$
Q t_{j}=\frac{\sum_{i=1}^{n t_{j}} Q_{i j}}{n t_{j}} \text { and } C T_{j}=\frac{\sum_{i=1}^{n t_{j}} P_{i j}}{n t_{j}} .
$$


TABLE 3. Notations.

\begin{tabular}{|c|l|}
\hline Variable & Description \\
\hline$m$ & CA agents number \\
\hline$C A_{1}, \ldots, C A_{m}$ & CA agents identifiers \\
\hline$H$ & Home node \\
\hline$W k_{i}$ & $\begin{array}{l}\text { Nodes sequence representing a } C A_{\mathrm{i}} \text { agent Workplan: } \\
\left(S_{i 1}, \ldots, S_{i p}\right) \text { with } 1 \leqslant p \leqslant J\end{array}$ \\
\hline$T\left(W k_{i}\right)$ & Routing time for $W k_{i}$ \\
\hline$Q t e_{k, u}$ & The size of the data transported by $C A_{k}$ from node $S_{u}$ \\
\hline $\operatorname{Tr}\left(Q t e_{k, u}, S_{u}, S_{u+1}\right)$ & Transmission time for $Q t e_{k, u}$ from node $S_{u}$ to node $S_{u+1}$ \\
\hline$C T_{i}$ & Processing time on node $S_{i}$ \\
\hline$Q t_{i}$ & Data quantity on node $S_{i}$ \\
\hline$L_{s}\left(S_{i}, S_{j}\right)$ & Shortest latency between nodes $S_{i}$ and $S_{j}$ \\
\hline
\end{tabular}

Definition 4. Qte $e_{k, u}$ (Data quantity transported from $S_{u}$ by $C A_{k}$ ) corresponds to collected data quantity by $C A_{k}$ during its route, until node $S_{u}$. It is computed by:

$$
Q t e_{k, u}=Q_{0}+\sum_{r=1}^{u} Q t_{r}
$$

Definition 5. $\operatorname{Tr}\left(Q t e_{k, u}, S_{u}, S_{u+1}\right)$ (Transmission time) needed time for the agent $C A_{k}$ to migrate from $S_{u}$ to $S_{u+1}$ transporting the data quantity $\mathrm{Qte}_{k, u}$. It is computed as follows:

$$
\operatorname{Tr}\left(\mathrm{Qte}_{k, u}, S_{u}, S_{u+1}\right)=\frac{\mathrm{Qte}_{k, u}}{\mathrm{Qte}_{\text {paquet } R}} \times L_{s}\left(S_{u}, S_{u+1}\right) .
$$

Qte $_{\text {paquet } R}$ is the size of a data packet on the current network $R$.

Definition 6. $T\left(W k_{k}\right)$ (Routing time for $W k_{k}$ ) needed time for the agent $C A_{k}$ to visit the sequence of network nodes $\left(S_{k 1}, \ldots, S_{k p}\right)$ with $1 \leqslant p \leqslant J$. Routing time is computed as follows:

$$
T\left(W k_{k}\right)=T_{\text {go }}+T_{\text {travel }}+T_{\text {return }} .
$$

\begin{tabular}{|c|c|c|c|c|}
\hline$p$ & $W k_{k}$ & $T_{\text {go }}$ & $T_{\text {return }}$ & $T_{\text {travel }}$ \\
\hline 1 & $\left(S_{k 1}\right)$ & \multirow{2}{*}{$\operatorname{Tr}\left(Q_{0}, H, S_{k 1}\right)$} & $\operatorname{Tr}\left(Q_{0}+Q t_{k 1}, S_{k 1}, H\right)$ & $C T_{1}$ \\
\cline { 4 - 5 } & & $\operatorname{Tr}\left(Q_{0}+Q t e_{k, p}, S_{k p}, H\right)$ & $X_{k}$ \\
\hline $1<p \leqslant J$ & $\left(S_{k 1}, \ldots, S_{k p}\right)$ & &
\end{tabular}

With:

$$
X_{k}=\sum_{i=1}^{p} C T_{k i}+\sum_{i=1}^{p-1} \operatorname{Tr}\left(Q t e_{k, i}, S_{k i}, S_{k i+1}\right)
$$


TABle 4. Example of available services (Processing time and data size).

\begin{tabular}{|c|c|c|c|c|}
\hline & $S_{1}$ & $S_{2}$ & $S_{3}$ & $S_{4}$ \\
\hline$T_{1}$ & $(0,0)$ & $(2,3)$ & $(4,3)$ & $(2,3)$ \\
\hline$T_{2}$ & $(2,5)$ & $(1,2)$ & $(4,1)$ & $(3,3)$ \\
\hline$T_{3}$ & $(1,3)$ & $(0,0)$ & $(2,3)$ & $(4,2)$ \\
\hline$T_{4}$ & $(3,1)$ & $(0,0)$ & $(0,0)$ & $(0,0)$ \\
\hline$T_{5}$ & $(2,1)$ & $(1,3)$ & $(4,2)$ & $(4,3)$ \\
\hline
\end{tabular}

\section{Proposed Workplan scheme}

To propose a cost-effective Workplan MA scheme, we assume that a monitoring module exists in the system providing information about network status (latency, bandwidth, traffic, bottleneck, failure ...). Therefore, we can get latencies values between all pairs of nodes on the MN. Latencies vary according to network state and are related to the data flow. We assume that MAs are created only in the Home node and are not cloned in other nodes as was done in [11]. It is clear that sending a MA to each node gives us the best total computing time because, in this case, agents are launched simultaneously into each network node. Our goal is to keep this best total computation time to built nodes partitions, minimizing the number of MAs. A well known Travelling Salesman Problem (TSP) algorithm [17] must be applied to each partition in order to optimize each agent local routing path. To resolve the same problem, Baek proposed a cost-effective MA planning algorithm [16]. We adopt the same approach considering data transmission through the network. In current section, we aim to find a set of CA agents minimizing their navigation time in order to explore all MN nodes, taking into account network latency. Consequently, we do not care about data cost (see Sect. 2). In this case, the service table will not express information cost of a service on a node. So the new service table presented by Table 4 below, will just express processing time and data size $\left(P_{i j}, Q_{i j}\right)$.

\section{The algorithm description}

As described previously, $L_{s}\left(S_{i}, S_{j}\right)$ namely shortest latency between two network nodes $S_{i}$ and $S_{j}$, is available. The algorithm is described as follow:

Step 1. Sort nodes in decreasing order according to their correspondent routing time $T\left(W k_{i}=S_{i}\right) \forall 1 \leqslant i \leqslant J$. Set the threshold $\delta$ which is the routing time of the first node in the sorted list:

$$
\delta=\max _{1 \leqslant i \leqslant J}\left(T\left(W k_{i}=S_{i}\right)\right)
$$


Step 2. Partition the given network into several parts by gathering nodes so that the routing time of each part does not exceed the threshold $\delta$.

Step 3. Run a TSP algorithm to optimize each routing path.

Step 4. Allocate a CA agent for each Workplan in order to explore the correspondent network partition.

We propose a dynamic algorithm which tries to find the next node from the current node where the agent resides. In other words, this algorithm looks for the next node for a part calculating new routing time. A node is selected if the new routing time does not exceed the threshold $\delta$. Otherwise, a Workplan is ready to be assigned to a CA and the algorithm ends if each MN node belongs to a routing path.

The algorithm above disperses all nodes into a set of CA agents in order to explore the MN totally. This step fixes the needed number $m$ of CA agents and organizes their initial Workplans. In the next section, a sub-set S' of MN will be identified thanks to an evolutionary method, in order to optimize the computing flow management. Therefore, some nodes may not be selected, what risks to modify the number of CA agents. It is where all nodes composing a Workplan of a $\mathrm{CA}$ agent, are not selected. In this case, the number of CA agents is reduced. The goal is to optimize the selection of the nodes from the set of information providers proposing the required customers services. Final Workplans will be deduced from initial ones in order to collect needed data satisfying users' requests as soon as possible with reasonable costs. Let $m^{\prime}$ be the new number of CA agents. We have also $J^{\prime}=\left|S^{\prime}\right|$ the new number of nodes so $m^{\prime} \leqslant m, J^{\prime} \leqslant J$ and $S^{\prime} \leqslant S$.

\section{DATA FLOW OPTIMISATION}

Evolutionary algorithms (EA), inspired from genetic algorithms, added a new aspect to the field of artificial intelligence. These algorithms use various computational models of evolutionary processes to solve problems on a computer. EA are stochastic search methods that mimic the metaphor of natural biological evolution; they operate on a population of potential solutions applying principle of survival of the fittest, to produce successively better approximations to a solution. At each generation, a new set of approximations is created by the process of selecting individuals according to their level of fitness in the population, then breeding them together using genetic operators such as crossover and mutation [18]. Compared to traditional optimization methods such as gradient descent, EA are robust and global search technique. For this reason, the scheduling community has been quick to realize the potential of EA. In this section [19], we consider the important characteristics of EA and their relevance for solving the traditional NP-complete problem. Therefore, some points must be clarified:

- A specific genetic representation (or encoding) appropriate to the problem, to determine feasible solutions of the scheduling optimization problem, 


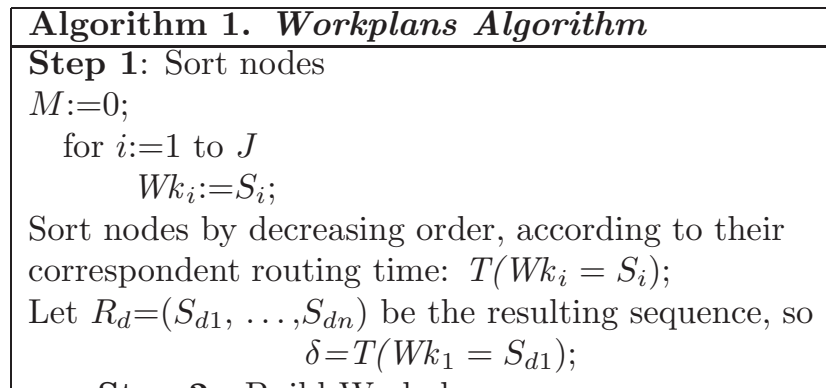

Step 2 : Build Workplans

$$
\text { for } j:=1 \text { to } J
$$

$W k_{j}=\varnothing$;

If $\exists$ "unassigned" $S_{d k}$ where $k$ is minimum

\{

Select it;

$m:=m+1$;

Union $\left(W k_{j}, S_{d k}\right)$;

Mark $S_{d k}$ as "assigned";

\}//end if

else terminate; // end step2

while (true)

\{

Sort nodes according to $L\left(S_{d k}, S_{d l}\right)$ with

$1<l \leqslant J$ and

$\mathrm{S}_{d l}$ is "unassigned" by increasing order

Let $\left(S_{e 1}, \ldots, S_{e g}\right)$ be the resulting sequence;

previous_k $:=k$;

for $y:=1$ to $g$

\{

Union $\left(W k_{j}, S_{e y}\right)$;

if $T\left(W k_{j}\right) \leqslant \delta$

\{

mark $S_{e y}$ as "assigned";

find $\mathrm{k}$ while $d k=e y$;

break for-loop;

\}//end if

else $W k_{j}:=W k_{j}-\left\{S_{e y}\right\}$;

\}//end inner for-loop

if previous_ $k=k$

break while-loop;

\}// end while-loop

\}// end outer for-loop

Step 3 : Run a simple TSP algorithm to optimize each $W k_{k} \quad \forall 1 \leqslant k \leqslant m$. 
- Original genetic operators that alter the composition of children during reproduction. As it was mentioned previously, a task (sub-request) must be managed by only one provider, selected from the set of nodes which propose the correspondent service (see Sect. 2). Therefore, we choose to correct generated solutions in order to respect this constraint. Consequently, each crossover or mutation operation must be followed by a correction process,

- Parents are selected randomly from current population to crossover with some probability $\mathrm{p}_{c}\left(0<p_{c}<1\right)$. We believe that this technique gives more chance to weak individuals to survey with minimum computation time,

- A non-elitist replacement technique is adopted to generate the new population from the previous one,

- The evaluation function estimates a possible solution according to two criteria: the cost and the delay (Sect. 5).

\section{A. Representation of a solution}

The selection of an appropriate representational scheme of a solution is fundamental to the success of EA applications. That's why we try to find an efficient coding for the chromosome respecting our problem constraints. Therefore, we propose a flexible representation of the chromosome called Flexible Tasks Assignment Representation (FeTAR). The chromosome is represented by a matrix $\mathrm{CH}\left(I^{\prime} \times J^{\prime}\right)$ where rows represent independent tasks, composing globally simultaneous requests and columns represent nodes. Each element of the matrix specifies the assignment of a node $\mathrm{S}_{j}$ to the task $\mathrm{T}_{i}$ as follow:

$$
\mathrm{CH}[i, j]=\left\{\begin{array}{l}
\mathbf{1 :} \text { if } S_{j} \text { is assigned to } T_{i} \\
*: \text { if } S_{j} \text { may be assigned to } T_{i} \\
\mathbf{X}:: \text { if } S_{j} \text { cannot be assigned to } T_{i} .
\end{array}\right.
$$

We notice that each task must be assigned, so we assume that each task must be performed at least by a node, selected from a set of nodes proposing the service which corresponds to a response to the concerned task.

Example. We consider 4 nodes and 3 requests, decomposed globally into 5 tasks as follow:

req $q_{1}$ decomposed into $T_{1}, T_{2}, T_{3}$ and $T_{5}$

req $q_{2}$ decomposed into $T_{2}$

req $q_{3}$ decomposed into $T_{1}, T_{3}$ and $T_{4}$ 
A possible solution has a $F e T A R$ scheme, specified as follows:

\begin{tabular}{|l|l|l|l|l|}
\hline $\mathrm{CH}$ & $S_{1}$ & $S_{2}$ & $S_{3}$ & $S_{4}$ \\
\hline$T_{1}$ & $\mathrm{X}$ & 1 & $*$ & $*$ \\
\hline$T_{2}$ & $*$ & $*$ & 1 & $*$ \\
\hline$T_{3}$ & 1 & $\mathrm{X}$ & $*$ & $*$ \\
\hline$T_{4}$ & 1 & $\mathrm{X}$ & $\mathrm{X}$ & $\mathrm{X}$ \\
\hline$T_{5}$ & $*$ & $*$ & 1 & $*$ \\
\hline
\end{tabular}

\section{B. Selection}

Selection is one of the most important aspects of all EA. It determines which individuals in the population will have all or some of its genetic material passed on the next generations. The objective of the selection method employed in EA, is to give polynomial increasing trials to fittest individuals. We use random technique, to give chance to weak individuals to survey: parents are selected randomly from current population to crossover with some probability $p_{c}\left(0<p_{c}<1\right)$.

\section{Genetic operators}

\section{Crossover operator}

Crossover involves combining elements from two parent chromosomes into one or more child chromosomes. The role of the crossover is to generate a better solution by exchanging information contained in the current good one [18]. We propose a single crossover operator presented by the following algorithm:

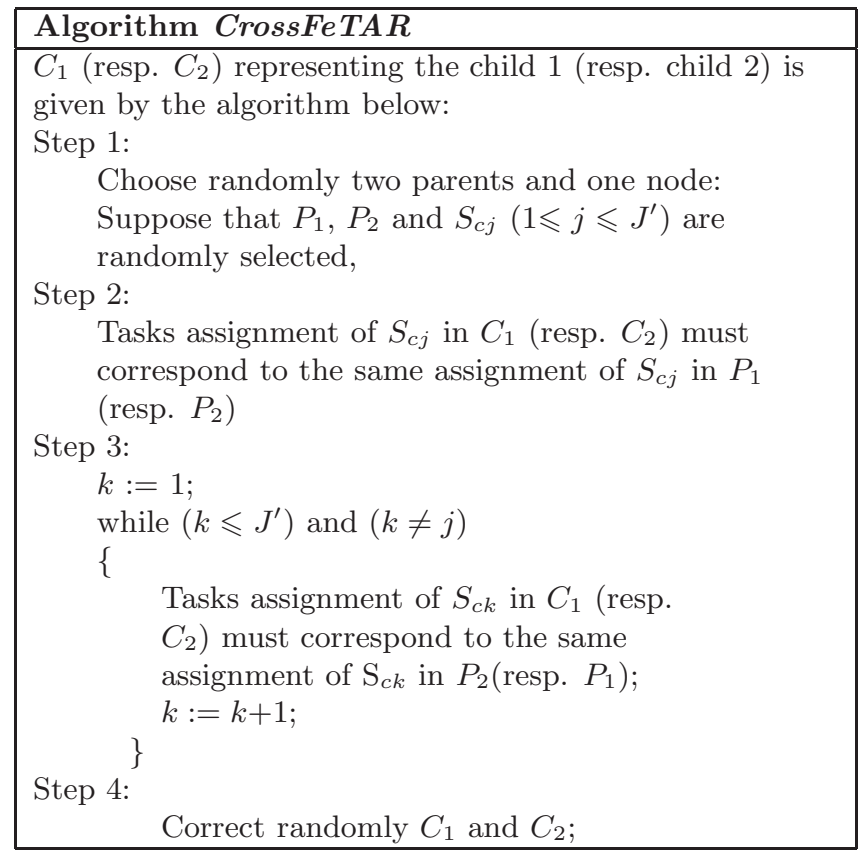


Crossover operator is illustrated by the example below:

\section{Example of crossover}

Step 1. Assume that $P_{1}, P_{2}$ and $S_{2}$ represent respectively two chromosomes and one node, randomly selected. $S_{2}$ corresponds to the second column of the matrix representing the chromosome:

\section{Parent 1}

\begin{tabular}{|c|c|c|c|c|}
\hline$P_{1}$ & $S_{1}$ & $S_{2}$ & $S_{3}$ & $S_{4}$ \\
\hline$T_{1}$ & $\mathrm{X}$ & 1 & $*$ & $*$ \\
\hline$T_{2}$ & $*$ & $*$ & 1 & $*$ \\
\hline$T_{3}$ & 1 & $\mathrm{X}$ & $*$ & $*$ \\
\hline$T_{4}$ & 1 & $\mathrm{X}$ & $\mathrm{X}$ & $\mathrm{X}$ \\
\hline$T_{5}$ & $*$ & $*$ & 1 & $*$ \\
\hline
\end{tabular}

Parent 2

\begin{tabular}{|c|c|c|c|c|}
\hline$P_{2}$ & $S_{1}$ & $S_{2}$ & $S_{3}$ & $S_{4}$ \\
\hline$T_{1}$ & $\mathrm{X}$ & $*$ & $*$ & 1 \\
\hline$T_{2}$ & $*$ & $*$ & 1 & $*$ \\
\hline$T_{3}$ & $*$ & $\mathrm{X}$ & 1 & $*$ \\
\hline$T_{4}$ & 1 & $\mathrm{X}$ & $\mathrm{X}$ & $\mathrm{X}$ \\
\hline$T_{5}$ & 1 & $*$ & $*$ & $*$ \\
\hline
\end{tabular}

Step 2. Tasks assignment of $S_{2}$ in $C_{1}$ (resp. $\mathrm{C}_{2}$ ) must corresponds to the same assignment of $\mathrm{S}_{2}$ in $\mathrm{P}_{1}$ (resp. $P_{2}$ )

Child 1

\begin{tabular}{|c|c|c|c|c|}
\hline$C_{1}$ & $S_{1}$ & $S_{2}$ & $S_{3}$ & $S_{4}$ \\
\hline$T_{1}$ & & 1 & & \\
\hline$T_{2}$ & & $*$ & & \\
\hline$T_{3}$ & & $\mathrm{X}$ & & \\
\hline$T_{4}$ & & $\mathrm{X}$ & & \\
\hline$T_{5}$ & & $*$ & & \\
\hline
\end{tabular}

Child 2

\begin{tabular}{|c|c|c|c|c|}
\hline$C_{2}$ & $S_{1}$ & $S_{2}$ & $S_{3}$ & $S_{4}$ \\
\hline$T_{1}$ & & $*$ & & \\
\hline$T_{2}$ & & $*$ & & \\
\hline$T_{3}$ & & $\mathrm{X}$ & & \\
\hline$T_{4}$ & & $\mathrm{X}$ & & \\
\hline$T_{5}$ & & $*$ & & \\
\hline
\end{tabular}


Step 3. Tasks assignment of $\mathrm{S}_{1}, S_{3}$ and $S_{4}$ in $C_{1}$ (resp. $C_{2}$ ) must corresponds to the same assignment of $S_{1}, S_{3}$ and $S_{4}$ in $P_{2}$ (resp. $P_{1}$ )

Child 1

\begin{tabular}{|c|c|c|c|c|}
\hline$C_{1}$ & $S_{1}$ & $S_{2}$ & $S_{3}$ & $S_{4}$ \\
\hline$T_{1}$ & $\mathrm{X}$ & $*$ & $*$ & 1 \\
\hline$T_{2}$ & $*$ & 1 & 1 & $*$ \\
\hline$T_{3}$ & $*$ & $\mathrm{X}$ & 1 & $*$ \\
\hline$T_{4}$ & 1 & $*$ & $\mathrm{X}$ & $\mathrm{X}$ \\
\hline$T_{5}$ & 1 & $*$ & $*$ & $*$ \\
\hline
\end{tabular}

Child 2

\begin{tabular}{|c|c|c|c|c|}
\hline$C_{2}$ & $S_{1}$ & $S_{2}$ & $S_{3}$ & $S_{4}$ \\
\hline$T_{1}$ & $\mathrm{X}$ & 1 & $*$ & $*$ \\
\hline$T_{2}$ & $*$ & $*$ & 1 & $*$ \\
\hline$T_{3}$ & 1 & $\mathrm{X}$ & $*$ & $*$ \\
\hline$T_{4}$ & 1 & $\mathrm{X}$ & $\mathrm{X}$ & $\mathrm{X}$ \\
\hline$T_{5}$ & $*$ & $*$ & 1 & $*$ \\
\hline
\end{tabular}

Step 4. $C_{1}$ is not a feasible solution contrary to $C_{2}$ because $C_{1}$ assigns task $T_{2}$ more than one time (in $C_{1}, S_{2}$ and $S_{3}$ are assigned simultaneously to $T_{2}$ ). After correction, $C_{1}$ will be randomly:

\begin{tabular}{|c|c|c|c|c|}
\hline$C_{1}$ & $S_{1}$ & $S_{2}$ & $S_{3}$ & $S_{4}$ \\
\hline$T_{1}$ & $\mathrm{X}$ & $*$ & $*$ & 1 \\
\hline$T_{2}$ & $*$ & 1 & $*$ & $*$ \\
\hline$T_{3}$ & $*$ & $\mathrm{X}$ & 1 & $*$ \\
\hline$T_{4}$ & 1 & $*$ & $\mathrm{X}$ & $\mathrm{X}$ \\
\hline$T_{5}$ & 1 & $*$ & $*$ & $*$ \\
\hline
\end{tabular}

$\mathrm{Or}$

\begin{tabular}{|c|c|c|c|c|}
\hline$C_{1}$ & $S_{1}$ & $S_{2}$ & $S_{3}$ & $S_{4}$ \\
\hline$T_{1}$ & $\mathrm{X}$ & $*$ & $*$ & 1 \\
\hline$T_{2}$ & $*$ & $*$ & 1 & $*$ \\
\hline$T_{3}$ & $*$ & $\mathrm{X}$ & 1 & $*$ \\
\hline$T_{4}$ & 1 & $*$ & $\mathrm{X}$ & $\mathrm{X}$ \\
\hline$T_{5}$ & 1 & $*$ & $*$ & $*$ \\
\hline
\end{tabular}

\section{Mutation operator}

Mutation represents another important genetic operator. Although mutation is important, it is secondary to crossover .It introduces some extra variability into 
the population and typically works with a single chromosome to create a new modified one [18]. The mutation algorithm is presented as follow:

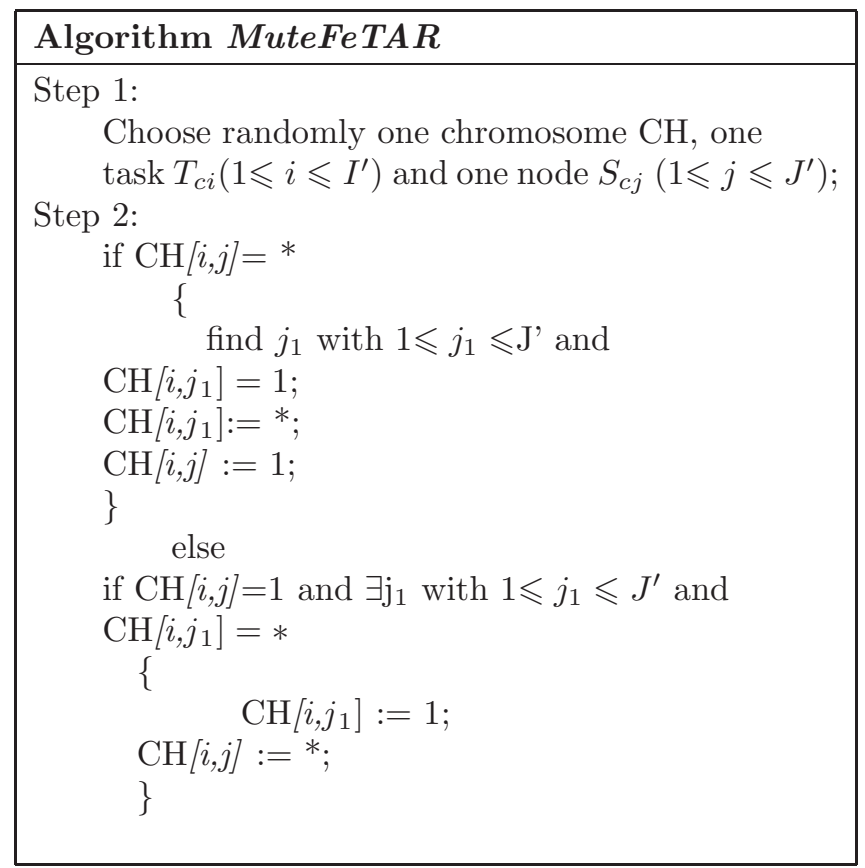

Mutation operator is illustrated by the example below:

\section{Example of mutation}

We use the same data of the example presented previously:

Step 1. Choose randomly one chromosome, one task and one node. We assume that the chromosome $\mathrm{CH}$, the task $T_{3}$ and the node $S_{1}$ are randomly selected:

Chromosome
\begin{tabular}{|c|c|c|c|c|}
\hline $\mathrm{CH}$ & $S_{1}$ & $S_{2}$ & $S_{3}$ & $S_{4}$ \\
\hline$T_{1}$ & $\mathrm{X}$ & 1 & $*$ & $*$ \\
\hline$T_{2}$ & $*$ & $*$ & 1 & $*$ \\
\hline$T_{3}$ & 1 & $\mathrm{X}$ & $*$ & $*$ \\
\hline$T_{4}$ & 1 & $\mathrm{X}$ & $\mathrm{X}$ & $\mathrm{X}$ \\
\hline$T_{5}$ & $*$ & $*$ & 1 & $*$ \\
\hline
\end{tabular}


Step 2. $\mathrm{CH}[3,1]=1$ and $\exists j_{1}$ (3 or 4$)$ with $\mathrm{CH}\left[3, j_{1}\right]={ }^{*}$ so replace $\mathrm{CH}[3,1]$ with *, choose randomly $j_{1}$ (3 for example) and finally replace $\mathrm{CH}[3,3]$ with 1 . New chromosome is presented as follow:

New Chromosome

\begin{tabular}{|c|c|c|c|c|}
\hline $\mathrm{CH}$ & $S_{1}$ & $S_{2}$ & $S_{3}$ & $S_{4}$ \\
\hline$T_{1}$ & $\mathrm{X}$ & 1 & $*$ & $*$ \\
\hline$T_{2}$ & $*$ & $*$ & 1 & $*$ \\
\hline$T_{3}$ & $*$ & $\mathrm{X}$ & 1 & $*$ \\
\hline$T_{4}$ & 1 & $\mathrm{X}$ & $\mathrm{X}$ & $\mathrm{X}$ \\
\hline$T_{5}$ & $*$ & $*$ & 1 & $*$ \\
\hline
\end{tabular}

\section{Evaluation FunCtions}

At each iteration, individuals (chromosomes) in the current population are evaluated according to the same measure of fitness. There is a number of characteristics of the evaluation function that enhance or hinder the evaluation of a program performance $[18,20]$. In our case, the fitness function intends to maximize the number of satisfied transport travellers, minimizing response delay and total cost. In other words, a chromosome is firstly evaluated according to the number of responses which respect due dates, namely responses minimizing correspondent ending dates and respecting correspondent due dates. Then a solution is evaluated according to its cost. Therefore, a chromosome has to express ending responses date and the information cost. To illustrate the evaluation of individuals in a population, we keep the same example previously presented: 4 nodes and 3 requests decomposed globally into 5 tasks as follow:

$$
\begin{aligned}
& R e q_{1} \text { decomposed into } T_{1}, T_{2}, T_{3} \text { and } T_{5} \mid d_{1}=10 \\
& R e q_{2} \text { decomposed into } T_{2} \mid d_{2}=5 \\
& R e q_{3} \text { decomposed into } T_{1}, T_{3} \text { and } T_{4} \mid d_{3}=10
\end{aligned}
$$

A same node (information provider) may propose different services and a same service may be proposed indifferently by several nodes. In current section, we aim to minimize processing time on MN nodes, reducing collected data cost in order to optimize the data flow management. Consequently, we do not care about collected data quantity (Sect. 2). In that follows, a service table will not express data size of a service on a node. So the new service table presented by Table 5 below, expresses the processing time and the cost $\left(P_{i j}, C_{i j}\right)$. 
TABle 5. Example of available services (Processing time and cost).

\begin{tabular}{|c|c|c|c|c|}
\hline & $S_{1}$ & $S_{2}$ & $S_{3}$ & $S_{4}$ \\
\hline$T_{1}$ & $\mathrm{X}$ & $*$ & $*$ & $*$ \\
\hline$T_{2}$ & $*$ & $*$ & $*$ & $*$ \\
\hline$T_{3}$ & $*$ & $\mathrm{X}$ & $*$ & $*$ \\
\hline$T_{4}$ & $*$ & $\mathrm{X}$ & $\mathrm{X}$ & $\mathrm{X}$ \\
\hline$T_{5}$ & $*$ & $*$ & $*$ & $*$ \\
\hline
\end{tabular}

FeTAR design corresponding to the service table below (Tab. 5) is:

\begin{tabular}{|c|c|c|c|c|}
\hline & $S_{1}$ & $S_{2}$ & $S_{3}$ & $S_{4}$ \\
\hline$T_{1}$ & $(0,0)$ & $(2,5)$ & $(4,3)$ & $(2,5)$ \\
\hline$T_{2}$ & $(2,4)$ & $(1,5)$ & $(4,5)$ & $(3,8)$ \\
\hline$T_{3}$ & $(1,7)$ & $(0,0)$ & $(2,5)$ & $(4,2)$ \\
\hline$T_{4}$ & $(3,2)$ & $(0,0)$ & $(0,0)$ & $(0,0)$ \\
\hline$T_{5}$ & $(2,3)$ & $(1,1)$ & $(4,5)$ & $(4,5)$ \\
\hline
\end{tabular}

A possible solution, instance of the FeTAR is:

\begin{tabular}{|c|c|c|c|c|}
\hline & $S_{1}$ & $S_{2}$ & $S_{3}$ & $S_{4}$ \\
\hline$T_{1}$ & $\mathrm{X}$ & 1 & $*$ & $*$ \\
\hline$T_{2}$ & $*$ & $*$ & 1 & $*$ \\
\hline$T_{3}$ & 1 & $\mathrm{X}$ & $*$ & $*$ \\
\hline$T_{4}$ & 1 & $\mathrm{X}$ & $\mathrm{X}$ & $\mathrm{X}$ \\
\hline$T_{5}$ & $*$ & $*$ & $*$ & 1 \\
\hline
\end{tabular}

A request $r e q_{w}$ is decomposed into $I_{t, w}$ tasks. Therefore, the total processing time EndReq $q_{w}$ for each $r e q_{w}$ is computed by the means of the algorithm fitness_1 below. This time includes only the effective processing time on the MN. We assume that, the ending date $D_{w}$ corresponding to the total execution time of a request $r e q_{w}$, includes also the average navigation time of CA agents (Sect. 3). This is expressed by:

$$
\begin{gathered}
\gamma=\delta-\frac{\sum_{j=1}^{J} C T_{j}}{J} \\
\Rightarrow \quad \forall 1 \leqslant w \leqslant R, D_{w}=\operatorname{EndReq}_{w}+\gamma
\end{gathered}
$$




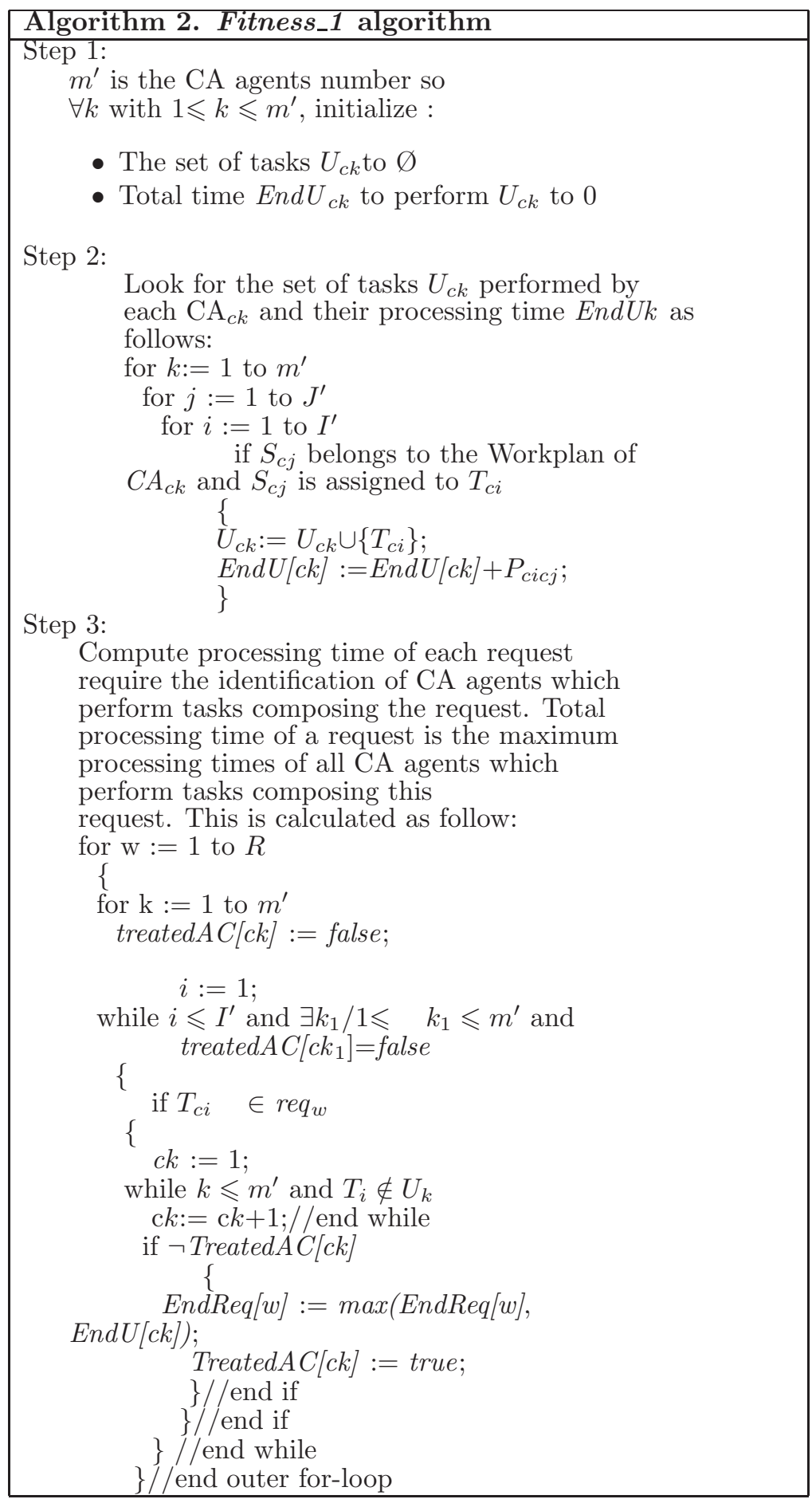


Total cost of a request $r e q_{w}$ is CostReq[w] expressed by $C_{w}$, is given by the mean of the algorithm below:

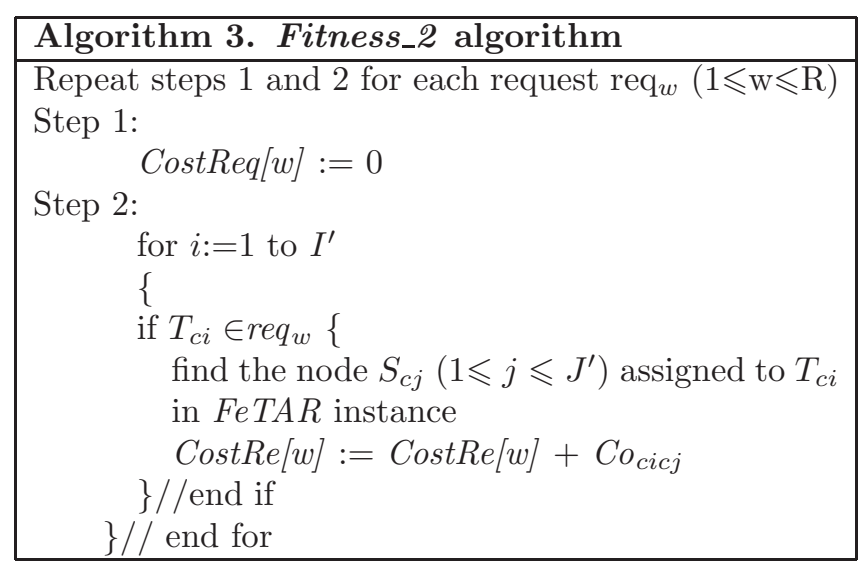

Knowing that using expression (1), we can deduce ending date from fitness_1 algorithm, the new FeTAR representation of the chromosome express for each request $\operatorname{req}_{w} 1 \leqslant \mathrm{w} \leqslant \mathrm{R}$, its ending date and its cost; it is illustrated by the example below:

\begin{tabular}{|c|c|c|c|c|c|c|c|c|}
\hline & $S_{1}$ & $S_{2}$ & $S_{3}$ & $S_{4}$ & $w$ & $d_{w}$ & $C_{w}$ & $D_{w}$ \\
\hline$T_{1}$ & $\mathrm{X}$ & 1 & $*$ & $*$ & 1 & 10 & 5 & 6 \\
\hline$T_{2}$ & $*$ & $*$ & 1 & $*$ & 2 & 5 & 1 & 1 \\
\hline$T_{3}$ & 1 & $\mathrm{X}$ & $*$ & $*$ & 3 & 10 & 4 & 2 \\
\hline$T_{4}$ & 1 & $\mathrm{X}$ & $\mathrm{X}$ & $\mathrm{X}$ & & & & \\
\hline$T_{5}$ & $*$ & $*$ & $*$ & 1 & & & & \\
\hline
\end{tabular}

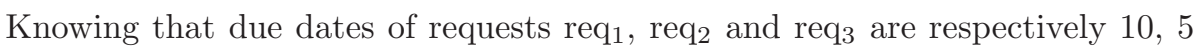
and 10, the solution satisfy all requests at latest in 6 units of time and has an average cost equals to 2.66 units of cost.

\section{E. Evolutionary APPROACH}

To determinate best solutions, we adopt an elitist approach [22] using an external storage to memorise the most adapted individuals during the search. The evolutionary adopted approach is shown by the diagram below (Fig. 5). During the evaluation process to crossover and mutation, best solutions are saved in external archives.

Knowing that $d_{\max }=\max \left(d_{w}\right)_{1 \leqslant w \leqslant R}$, we discern two archive sets:

- Main solutions archive $(M)$ representing best solutions which respect all due dates. In other words, a chromosome $\mathrm{CH} \in \mathrm{M}$ if and only if $\forall 1 \leqslant w \leqslant R$, $D_{w} \leqslant d_{\max }$. this archive set is decomposed into two sub-archives:

$\circ$ dominant solutions archive $(d)$;

○ $\varepsilon$-dominant solutions archive $(\varepsilon-d)$. 


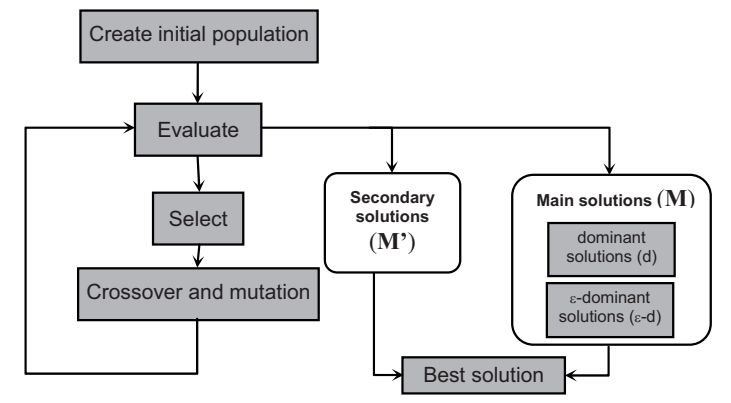

FiguRE 5. Evolutionary approach.

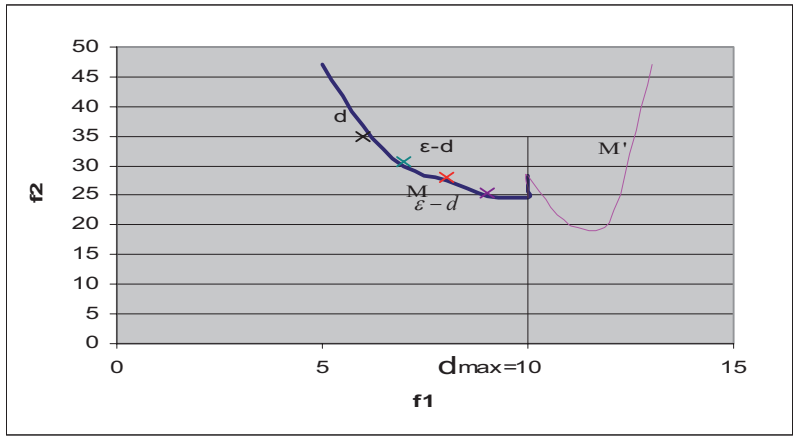

Figure 6. Optimal fronts.

- Secondary solutions archive $\left(M^{\prime}\right)$ representing best solutions which exceed at least one due date. In other words, a chromosome $\mathrm{CH} \in M^{\prime}$ if and only if $\exists w, 1 \leqslant w \leqslant R$ and $d_{\max }<D_{w}$.

We discerned two archive sets according to delay criterion satisfaction because we assume that if a response exceeds its due date, the user is not satisfied. That's why we consider that the first criterion has more priority that the second one. Consequently archive sets are firstly sorted according to delay criterion, then according to cost criterion. We notice by $\mathrm{f} 1$ the response delay function evaluated by fitness_1 algorithm and by f2 the cost function evaluated by fitness_2 algorithm (Sect. 5). Considering two different solutions $\mathrm{CH}$ and $\mathrm{CH}$ ', if $\mathrm{CH} \in \mathrm{M}$ and $\mathrm{CH}^{\prime} \in \mathrm{M}^{\prime}$ then $\mathrm{CH}$ dominates $\mathrm{CH}$ '. Otherwise, $\mathrm{CH}$ dominates $\mathrm{CH}^{\prime}$ if and only if $\mathrm{f} 1(\mathrm{CH})=\mathrm{f} 1\left(\mathrm{CH}^{\prime}\right)$ and $\mathrm{f} 2(\mathrm{CH})<\mathrm{f} 2\left(\mathrm{CH}^{\prime}\right)$.

Each archive set has a maximum number size equals to the population size. If the number of individuals in an archive exceeds this fixed size, a crowding process [27] must occur to decide which solutions must kept in the archive. The non selected solutions are deleted; and the others contribute to the next selection procedure; archive members can then transmit their characteristics to offspring 
TABLE 6. Requests decomposition.

\begin{tabular}{|l|l|l|l|l|}
\hline$w$ & 1 & 2 & 3 & 4 \\
\hline$T_{2}$ & & $\bullet$ & & \\
\hline$T_{3}$ & & $\bullet$ & $\bullet$ & \\
\hline$T_{5}$ & $\bullet$ & $\bullet$ & $\bullet$ & $\bullet$ \\
\hline$T_{6}$ & $\bullet$ & & & \\
\hline$T_{7}$ & & & $\bullet$ & $\bullet$ \\
\hline$T_{8}$ & $\bullet$ & $\bullet$ & $\bullet$ & \\
\hline$T_{9}$ & & $\bullet$ & & \\
\hline$T_{10}$ & & $\bullet$ & $\bullet$ & \\
\hline$T_{11}$ & $\bullet$ & $\bullet$ & $\bullet$ & \\
\hline$T_{12}$ & & & $\bullet$ & \\
\hline$T_{13}$ & $\bullet$ & & & $\bullet$ \\
\hline$T_{14}$ & & $\bullet$ & & \\
\hline$T_{15}$ & $\bullet$ & & $\bullet$ & \\
\hline & & & & \\
\hline$d_{w}$ & 1000 & 1000 & 1000 & 1000 \\
\hline
\end{tabular}

- Means that $T_{i}$ composes $r e q_{w}$

populations. $M$ and $M^{\prime}$ archive sets represent generated solutions having minimum $f_{1}$ and $f_{2}$ values, so if a chromosome $\mathrm{CH}$ of the offspring dominates any archive member $C H$ ', the archive member is deleted and the offspring is accepted. Figure 6 above represents the Pareto-optimal fronts with $\varepsilon_{1}=\varepsilon_{2}=\varepsilon=0.75$ [22]. We use a population size of $N=100$ with a crossover probability $p_{c}=0.8$ and mutation probability $p_{m}=0.2$.

\section{Simulation Results}

\subsection{NEEDED DATA}

\section{A. Requests Decomposition and due Dates}

We consider 4 requests decomposed globally into 13 tasks (see Tab. 6). We remind that $\mathrm{d}_{w}$ represents the due date of the request req $w(1 \leqslant w \leqslant 4)$. We have $I_{t}^{\prime} \subseteq I_{t}$, the set of tasks representing services composing simultaneous requests from the set of proposed services on the whole network.

\section{B. Shortest Latencies}

We consider 20 nodes representing information providers on the network. The latency range is $10 \mathrm{~ms} \sim 100 \mathrm{~ms}$. 
TABLE 7. Available services.

\begin{tabular}{|l|l|l|l|l|l|}
\hline & $S_{1}$ & $S_{2}$ & $S_{3}$ & $S_{4}$ & $S_{5}$ \\
\hline$T_{1}$ & $(8,2,5)$ & $(0,0,0)$ & $(5,3,10)$ & $(2,7,5)$ & $(10,2,3)$ \\
\hline$T_{2}$ & $(8,3,4)$ & $(0,0,0)$ & $(10,3,3)$ & $(3,7,6)$ & $(0,0,0)$ \\
\hline$T_{3}$ & $(8,5,4)$ & $(0,0,0)$ & $(8,3,2)$ & $(4,3,4)$ & $(4,10,4)$ \\
\hline$T_{4}$ & $(9,5,7)$ & $(2,5,4)$ & $(1,9,7)$ & $(2,7,8)$ & $(3,4,3)$ \\
\hline$T_{5}$ & $(8,10,3)$ & $(0,0,0)$ & $(2,6,7)$ & $(8,8,10)$ & $(8,2,10)$ \\
\hline$T_{6}$ & $(8,6,5)$ & $(0,0,0)$ & $(9,9,2)$ & $(8,2,9)$ & $(2,2,10)$ \\
\hline$T_{7}$ & $(0,0,0)$ & $(0,0,0)$ & $(6,7,1)$ & $(9,3,8)$ & $(0,0,0)$ \\
\hline$T_{8}$ & $(9,7,6)$ & $(0,0,0)$ & $(7,8,3)$ & $(1,7,3)$ & $(0,0,0)$ \\
\hline$T_{9}$ & $(0,0,0)$ & $(0,0,0)$ & $(0,0,0)$ & $(10,7,3)$ & $(0,0,0)$ \\
\hline$T_{10}$ & $(0,0,0)$ & $(0,0,0)$ & $(0,0,0)$ & $(9,4,5)$ & $(10,6,7)$ \\
\hline$T_{11}$ & $(0,0,0)$ & $(0,0,0)$ & $(10,6,7)$ & $(3,10,3)$ & $(10,9,8)$ \\
\hline$T_{12}$ & $(7,6,4)$ & $(0,0,0)$ & $(1,3,2)$ & $(9,8,2)$ & $(2,1,10)$ \\
\hline$T_{13}$ & $(1,7,4)$ & $(0,0,0)$ & $(9,6,5)$ & $(1,6,3)$ & $(3,4,2)$ \\
\hline$T_{14}$ & $(0,0,0)$ & $(0,0,0)$ & $(0,0,0)$ & $(2,10,8)$ & $(0,0,0)$ \\
\hline$T_{15}$ & $(3,7,8)$ & $(0,0,0)$ & $(10,7,4)$ & $(4,10,9)$ & $(8,8,7)$ \\
\hline
\end{tabular}

\begin{tabular}{|l|l|l|l|l|l|}
\hline & $S_{6}$ & $S_{7}$ & $S_{8}$ & $S_{9}$ & $S_{10}$ \\
\hline$T_{1}$ & $(9,9,6)$ & $(9,8,5)$ & $(1,10,6)$ & $(2,3,2)$ & $(9,9,8)$ \\
\hline$T_{2}$ & $(0,0,0)$ & $(4,9,6)$ & $(6,5,9)$ & $(4,6,3)$ & $(2,3,9)$ \\
\hline$T_{3}$ & $(0,0,0)$ & $(7,4,1)$ & $(8,6,5)$ & $(8,8,9)$ & $(6,8,9)$ \\
\hline$T_{4}$ & $(0,0,0)$ & $(3,7,10)$ & $(1,8,3)$ & $(10,10,9)$ & $(10,10,4)$ \\
\hline$T_{5}$ & $(0,0,0)$ & $(10,2,10)$ & $(4,9,3)$ & $(9,4,5)$ & $(3,6,6)$ \\
\hline$T_{6}$ & $(0,0,0)$ & $(2,8,7)$ & $(3,8,4)$ & $(2,7,1)$ & $(4,3,2)$ \\
\hline$T_{7}$ & $(0,0,0)$ & $(0,0,0)$ & $(7,9,3)$ & $(2,10,4)$ & $(0,0,0)$ \\
\hline$T_{8}$ & $(0,0,0)$ & $(7,1,3)$ & $(5,3,8)$ & $(2,6,9)$ & $(10,3,9)$ \\
\hline$T_{9}$ & $(0,0,0)$ & $(0,0,0)$ & $(3,10,3)$ & $(10,5,8)$ & $(1,1,3)$ \\
\hline$T_{10}$ & $(0,0,0)$ & $(0,0,0)$ & $(0,0,0)$ & $(9,8,10)$ & $(8,10,10)$ \\
\hline$T_{11}$ & $(0,0,0)$ & $(9,10,3)$ & $(7,2,3)$ & $(8,3,4)$ & $(3,2,9)$ \\
\hline$T_{12}$ & $(0,0,0)$ & $(0,0,0)$ & $(0,0,0)$ & $(0,0,0)$ & $(9,8,8)$ \\
\hline$T_{13}$ & $(0,0,0)$ & $(0,0,0)$ & $(8,7,2)$ & $(4,5,1)$ & $(9,5,8)$ \\
\hline$T_{14}$ & $(0,0,0)$ & $(10,6,1)$ & $(0,0,0)$ & $(0,0,0)$ & $(2,10,5)$ \\
\hline$T_{15}$ & $(0,0,0)$ & $(0,0,0)$ & $(2,8,5)$ & $(2,9,4)$ & $(8,1,1)$ \\
\hline
\end{tabular}

\section{Available services}

We have 20 information providers on the network proposing 15 services in different way as follows in Table 7 . 
TABle 7. Continued.

\begin{tabular}{|l|l|l|l|l|l|}
\hline & $S_{11}$ & $S_{12}$ & $S_{13}$ & $S_{14}$ & $S_{15}$ \\
\hline$T_{1}$ & $(7,4,4)$ & $(3,2,2)$ & $(0,0,0)$ & $(10,2,10)$ & $(7,10,1)$ \\
\hline$T_{2}$ & $(0,0,0)$ & $(10,10,5)$ & $(5,1,9)$ & $(1,1,8)$ & $(9,7,4)$ \\
\hline$T_{3}$ & $(4,6,9)$ & $(7,5,9)$ & $(0,0,0)$ & $(3,4,5)$ & $(5,1,8)$ \\
\hline$T_{4}$ & $(0,0,0)$ & $(0,0,0)$ & $(3,10,3)$ & $(8,7,2)$ & $(8,8,3)$ \\
\hline$T_{5}$ & $(5,6,2)$ & $(4,4,10)$ & $(0,0,0)$ & $(6,9,2)$ & $(0,0,0)$ \\
\hline$T_{6}$ & $(1,7,8)$ & $(4,8,10)$ & $(0,0,0)$ & $(0,0,0)$ & $(7,5,4)$ \\
\hline$T_{7}$ & $(0,0,0)$ & $(4,1,2)$ & $(8,8,8)$ & $(6,1,6)$ & $(5,2,1)$ \\
\hline$T_{8}$ & $(0,0,0)$ & $(10,6,9)$ & $(5,2,1)$ & $(5,2,10)$ & $(9,5,5)$ \\
\hline$T_{9}$ & $(10,4,2)$ & $(9,1,10)$ & $(5,6,2)$ & $(0,0,0)$ & $(8,8,4)$ \\
\hline$T_{10}$ & $(8,1,1)$ & $(0,0,0)$ & $(0,0,0)$ & $(10,3,2)$ & $(0,0,0)$ \\
\hline$T_{11}$ & $(0,0,0)$ & $(10,1,10)$ & $(0,0,0)$ & $(7,3,7)$ & $(3,9,1)$ \\
\hline$T_{12}$ & $(9,5,4)$ & $(0,0,0)$ & $(4,3,9)$ & $(0,0,0)$ & $(8,5,10)$ \\
\hline$T_{13}$ & $(4,8,3)$ & $(8,4,6)$ & $(0,0,0)$ & $(0,0,0)$ & $(7,2,6)$ \\
\hline$T_{14}$ & $(6,10,2)$ & $(3,3,2)$ & $(6,1,9)$ & $(5,2,3)$ & $(0,0,0)$ \\
\hline$T_{15}$ & $(3,4,6)$ & $(9,7,3)$ & $(2,2,6)$ & $(0,0,0)$ & $(7,2,9)$ \\
\hline \hline & $S_{16}$ & $S_{17}$ & $S_{18}$ & $S_{19}$ & $S_{20}$ \\
\hline$T_{1}$ & $(0,0,0)$ & $(2,6,8)$ & $(5,7,7)$ & $(0,0,0)$ & $(0,0,0)$ \\
\hline$T_{2}$ & $(0,0,0)$ & $(8,10,9)$ & $(5,1,6)$ & $(9,7,1)$ & $(3,6,4)$ \\
\hline$T_{3}$ & $(1,4,5)$ & $(0,0,0)$ & $(4,10,9)$ & $(4,1,4)$ & $(6,5,5)$ \\
\hline$T_{4}$ & $(1,10,10)$ & $(0,0,0)$ & $(0,0,0)$ & $(0,0,0)$ & $(5,10,6)$ \\
\hline$T_{5}$ & $(9,6,8)$ & $(4,4,2)$ & $(6,5,9)$ & $(6,6,10)$ & $(1,2,10)$ \\
\hline$T_{6}$ & $(10,3,7)$ & $(8,9,8)$ & $(5,2,7)$ & $(4,5,3)$ & $(10,2,3)$ \\
\hline$T_{7}$ & $(0,0,0)$ & $(0,0,0)$ & $(9,9,10)$ & $(10,5,6)$ & $(1,2,3)$ \\
\hline$T_{8}$ & $(5,5,7)$ & $(6,8,7)$ & $(1,2,9)$ & $(0,0,0)$ & $(2,6,5)$ \\
\hline$T_{9}$ & $(0,0,0)$ & $(1,4,8)$ & $(0,0,0)$ & $(0,0,0)$ & $(9,1,5)$ \\
\hline$T_{10}$ & $(4,1,8)$ & $(9,10,1)$ & $(0,0,0)$ & $(6,5,10)$ & $(0,0,0)$ \\
\hline$T_{11}$ & $(1,2,7)$ & $(9,1,10)$ & $(5,10,10)$ & $(7,3,6)$ & $(6,8,1)$ \\
\hline$T_{12}$ & $(5,10,3)$ & $(10,9,7)$ & $(9,8,8)$ & $(0,0,0)$ & $(6,10,9)$ \\
\hline$T_{13}$ & $(4,6,2)$ & $(0,0,0)$ & $(7,10,4)$ & $(9,7,5)$ & $(0,0,0)$ \\
\hline$T_{14}$ & $(10,5,1)$ & $(3,2,7)$ & $(6,1,1)$ & $(1,4,4)$ & $(3,8,7)$ \\
\hline$T_{15}$ & $(10,3,4)$ & $(0,0,0)$ & $(9,2,7)$ & $(7,5,9)$ & $(4,5,4)$ \\
\hline
\end{tabular}

From Table 7 , we can generate for each node $S_{j}(1 \leqslant j \leqslant J)$, values of $n t_{j}$ and $C T_{j}$ corresponding the number of available services and the average of processing time on each node: 


\begin{tabular}{|l|l|l|}
\hline$S_{j}$ & $n t_{j}$ & $C T_{j}$ \\
\hline$S_{1}$ & 10 & 6.9 \\
\hline$S_{2}$ & 1 & 2 \\
\hline$S_{3}$ & 12 & 6.5 \\
\hline$S_{4}$ & 15 & 5 \\
\hline$S_{5}$ & 10 & 6 \\
\hline$S_{6}$ & 1 & 9 \\
\hline$S_{7}$ & 9 & 6.778 \\
\hline$S_{8}$ & 12 & 4.583 \\
\hline$S_{9}$ & 13 & 5.538 \\
\hline$S_{10}$ & 14 & 6 \\
\hline$S_{11}$ & 10 & 5.7 \\
\hline$S_{12}$ & 12 & 6.75 \\
\hline$S_{13}$ & 8 & 4.75 \\
\hline$S_{14}$ & 10 & 6.1 \\
\hline$S_{15}$ & 12 & 6.916 \\
\hline$S_{16}$ & 11 & 5.45 \\
\hline$S_{17}$ & 10 & 6 \\
\hline$S_{18}$ & 12 & 5.916 \\
\hline$S_{19}$ & 10 & 6.3 \\
\hline$S_{20}$ & 12 & 4.666 \\
\hline
\end{tabular}

$$
\text { So } \frac{\sum_{j=1}^{20} C T_{j}}{20}=5.5 \mathrm{~ms} \text {. }
$$

\section{WORKPLANS}

Algorithm 1 presented in Section 4 generates 9 CAs Workplans to explore totally a MN composed of 20 nodes as follows:

$W k_{1}=\left(S_{18}, S_{13}, S_{12}\right), W k_{2}=\left(S_{5}, S_{20}, S_{1}, S_{4}\right)$

$W k_{3}=\left(S_{17}, S_{3}\right), W k_{4}=\left(S_{16}, S_{10}\right)$

$W k_{5}=\left(S_{2}, S_{8}\right), W k_{6}=\left(S_{9}, S_{11}\right)$

$W k_{7}=\left(S_{15}, S_{14}\right), W k_{8}=\left(S_{19}, S_{7}\right), W k_{9}=\left(S_{6}\right)$

With $\delta=539.8 \mathrm{~ms}$, so the average navigation time of CA agents is:

$$
\gamma=\delta-\frac{\sum_{j=1}^{20} C T_{j}}{20}=534.3 \mathrm{~ms} .
$$


According to Table 7 , nodes $S_{2}$ and $S_{6}$ do not propose any service corresponding to tasks which compose simultaneous requests so $J^{\prime}=18$. Therefore Workplans are updated as follows:

$$
\begin{aligned}
& \boldsymbol{W} \boldsymbol{k}_{1}=\left(\boldsymbol{S}_{18}, \boldsymbol{S}_{13}, \boldsymbol{S}_{12}\right), \boldsymbol{W} \boldsymbol{k}_{2}=\left(\boldsymbol{S}_{5}, \boldsymbol{S}_{20}, \boldsymbol{S}_{1}, \boldsymbol{S}_{4}\right) \\
& \boldsymbol{W} \boldsymbol{k}_{3}=\left(\boldsymbol{S}_{17}, \boldsymbol{S}_{3}\right), \boldsymbol{W} \boldsymbol{k}_{4}=\left(\boldsymbol{S}_{16}, \boldsymbol{S}_{10}\right) \\
& \boldsymbol{W} \boldsymbol{k}_{5}=\left(\boldsymbol{S}_{8}\right), \boldsymbol{W} \boldsymbol{k}_{6}=\left(\boldsymbol{S}_{9}, \boldsymbol{S}_{11}\right) \\
& \boldsymbol{W} \boldsymbol{k}_{7}=\left(\boldsymbol{S}_{15}, \boldsymbol{S}_{14}\right), \boldsymbol{W k _ { 8 }}=\left(\boldsymbol{S}_{19}, \boldsymbol{S}_{7}\right), \boldsymbol{W} \boldsymbol{k}_{9}=\emptyset
\end{aligned}
$$

We can see here that the number of CAs changes to $m^{\prime}=8$.

\subsection{Possible solution}

We adjusted our program parameters as follow:

Crossover probability $p_{c}=0.9$

Mutation probability $p_{m}=0.2$

Population size $=100$

Generations number $=5000$.

The main generated solutions archive is not empty. Therefore we select a solution $\mathrm{CH} \in M$ having the best delay response:

\begin{tabular}{|l|l|l|l|l|l|l|l|l|l|}
\hline & $S_{1}$ & $S_{3}$ & $S_{4}$ & $S_{5}$ & $S_{7}$ & $S_{8}$ & $S_{9}$ & $S_{10}$ & $S_{11}$ \\
\hline$T_{2}$ & $*$ & $*$ & $*$ & $\times$ & $*$ & $*$ & $*$ & $*$ & $\times$ \\
\hline$T_{3}$ & $*$ & $*$ & $*$ & $*$ & $*$ & $*$ & $*$ & $*$ & $*$ \\
\hline$T_{5}$ & $*$ & 1 & $*$ & $*$ & $*$ & $*$ & $*$ & $*$ & $*$ \\
\hline$T_{6}$ & $*$ & $*$ & $*$ & $*$ & 1 & $*$ & $*$ & $*$ & $*$ \\
\hline$T_{7}$ & $\times$ & $*$ & $*$ & $\times$ & $\times$ & $*$ & 1 & $\times$ & $\times$ \\
\hline$T_{8}$ & $*$ & $*$ & $*$ & $\times$ & $*$ & $*$ & $*$ & $*$ & $\times$ \\
\hline$T_{9}$ & $\times$ & $\times$ & $*$ & $\times$ & $\times$ & 1 & $*$ & $*$ & $*$ \\
\hline$T_{10}$ & $\times$ & $\times$ & $*$ & $*$ & $\times$ & $\times$ & $*$ & $*$ & $*$ \\
\hline$T_{11}$ & $\times$ & $*$ & $*$ & $*$ & $*$ & $*$ & $*$ & $*$ & $\times$ \\
\hline$T_{12}$ & $*$ & $*$ & $*$ & 1 & $\times$ & $\times$ & $\times$ & $*$ & $*$ \\
\hline$T_{13}$ & $*$ & $*$ & 1 & $*$ & $\times$ & $*$ & $*$ & $*$ & $*$ \\
\hline$T_{14}$ & $\times$ & $\times$ & $*$ & $\times$ & $*$ & $\times$ & $\times$ & $*$ & $*$ \\
\hline$T_{15}$ & $*$ & $*$ & $*$ & $*$ & $\times$ & 1 & $*$ & $*$ & $*$ \\
\hline
\end{tabular}




\begin{tabular}{|l|l|l|l|l|l|l|l|l|l|}
\hline & $S_{12}$ & $S_{13}$ & $S_{14}$ & $S_{15}$ & $S_{16}$ & $S_{17}$ & $S_{18}$ & $S_{19}$ & $S_{20}$ \\
\hline$T_{2}$ & $*$ & $*$ & 1 & $*$ & $\times$ & $*$ & $*$ & $*$ & $*$ \\
\hline$T_{3}$ & $*$ & $\times$ & $*$ & $*$ & $*$ & $\times$ & 1 & $*$ & $*$ \\
\hline$T_{5}$ & $*$ & $\times$ & $*$ & $\times$ & $*$ & $*$ & $*$ & $*$ & $*$ \\
\hline$T_{6}$ & $*$ & $\times$ & $\times$ & $*$ & $*$ & $*$ & $*$ & $*$ & $*$ \\
\hline$T_{7}$ & $*$ & $*$ & $*$ & $*$ & $\times$ & $\times$ & $*$ & $*$ & $*$ \\
\hline$T_{8}$ & $*$ & $*$ & $*$ & $*$ & $*$ & $*$ & 1 & $\times$ & $*$ \\
\hline$T_{9}$ & $*$ & $*$ & $\times$ & $*$ & $\times$ & $*$ & $\times$ & $\times$ & $*$ \\
\hline$T_{10}$ & $\times$ & $\times$ & $*$ & $\times$ & 1 & $*$ & $\times$ & $*$ & $\times$ \\
\hline$T_{11}$ & $*$ & $\times$ & $*$ & 1 & $*$ & $*$ & $*$ & $*$ & $*$ \\
\hline$T_{12}$ & $\times$ & $*$ & $\times$ & $*$ & $*$ & $*$ & $*$ & $\times$ & $*$ \\
\hline$T_{13}$ & $*$ & $\times$ & $\times$ & $*$ & $*$ & $\times$ & $*$ & $*$ & $\times$ \\
\hline$T_{14}$ & $*$ & $*$ & $*$ & $\times$ & $*$ & 1 & $*$ & $*$ & $*$ \\
\hline$T_{15}$ & $*$ & $*$ & $\times$ & $*$ & $*$ & $\times$ & $*$ & $*$ & $*$ \\
\hline
\end{tabular}

\subsection{Final WORKPLANS}

Nodes $S_{1}, S_{10}, S_{11}, S_{12}, S_{13}, S_{19}$ and $S_{20}$ were not selected to assign tasks. Our solution is converted into a FeTAR design with $I^{\prime}=13$ and $J^{\prime}=11$.

Final Workplans are:

$$
\begin{aligned}
& \mathbf{W k}_{1}=\left(\mathrm{S}_{18}\right) \text { to perform }\left\{\mathrm{T}_{3}, \mathrm{~T}_{8}\right\} \\
& \mathbf{W k}_{2}=\left(\mathrm{S}_{5}, \mathrm{~S}_{4}\right) \text { to perform } \\
& \left\{\mathrm{T}_{12}\right\} \text { on } \mathbf{S}_{5} \\
& \left\{\mathbf{T}_{13}\right\} \text { on } \mathbf{S}_{4} \\
& \mathbf{W k}_{3}=\left(\mathrm{S}_{17}, \mathbf{S}_{3}\right) \text { to perform } \\
& \left\{\mathbf{T}_{14}\right\} \text { on } \mathbf{S}_{17} \\
& \left\{\mathbf{T}_{5}\right\} \text { on } \mathbf{S}_{3} \\
& \mathbf{W k}_{4}=\left(\mathbf{S}_{16}\right) \text { to perform }\left\{\mathbf{T}_{10}\right\} \\
& \mathbf{W k}_{5}=\left(\mathbf{S}_{8}\right) \text { to perform }\left\{\mathbf{T}_{9}, \mathbf{T}_{15}\right\} \\
& \mathbf{W k}_{6}=\left(\mathbf{S}_{9}\right) \text { to perform }\left\{\mathbf{T}_{7}\right\} \\
& \mathbf{W k}_{7}=\left(\mathbf{S}_{15}, \mathbf{S}_{14}\right) \text { to perform } \\
& \left\{\mathbf{T}_{11}\right\} \text { on } \mathbf{S}_{15} \\
& \left\{\mathbf{T}_{2}\right\} \text { on } \mathbf{S}_{14} \\
& \mathbf{W k}_{8}=\left(\mathbf{S}_{7}\right) \text { to perform }\left\{\mathbf{T}_{6}\right\}
\end{aligned}
$$

\subsection{Fitness}

fitness_1 and fitness_2 algorithms (Sect. 5) evaluate selected solution as follow in Table 9.

We notice here that this solution satisfy all requests respecting due dates through $539.3 \mathrm{~ms}$ and costs 37.25 units of cost. In order to evaluate the efficiently of our proposed optimization approach, we propose to compare it to the classical client server (CS) paradigm. 
TABLE 8. Generated FeTAR instance.

\begin{tabular}{|c|c|c|c|c|c|c|c|c|c|c|c|}
\hline & $S_{3}$ & $S_{4}$ & $S_{5}$ & $S_{7}$ & $S_{8}$ & $S_{9}$ & $S_{14}$ & $S_{15}$ & $S_{16}$ & $S_{17}$ & $S_{18}$ \\
\hline$T_{2}$ & $*$ & $*$ & $\times$ & $*$ & $*$ & $*$ & 1 & $*$ & $\times$ & $*$ & $*$ \\
\hline$T_{3}$ & $*$ & $*$ & $*$ & $*$ & $*$ & $*$ & $*$ & $*$ & $*$ & $\times$ & 1 \\
\hline$T_{5}$ & 1 & $*$ & $*$ & $*$ & $*$ & $*$ & $*$ & $\times$ & $*$ & $*$ & $*$ \\
\hline$T_{6}$ & $*$ & $*$ & $*$ & 1 & $*$ & $*$ & $\times$ & $*$ & $*$ & $*$ & $*$ \\
\hline$T_{7}$ & $*$ & $*$ & $\times$ & $\times$ & $*$ & 1 & $*$ & $*$ & $\times$ & $\times$ & $*$ \\
\hline$T_{8}$ & $*$ & $*$ & $\times$ & $*$ & $*$ & $*$ & $*$ & $*$ & $*$ & $*$ & 1 \\
\hline$T_{9}$ & $\times$ & $*$ & $\times$ & $\times$ & 1 & $*$ & $\times$ & $*$ & $\times$ & $*$ & $\times$ \\
\hline$T_{10}$ & $\times$ & $*$ & $*$ & $\times$ & $\times$ & $\mathrm{D}$. & $*$ & $\times$ & 1 & $*$ & $\times$ \\
\hline$T_{11}$ & $*$ & $*$ & $*$ & $*$ & $*$ & $*$ & $*$ & 1 & $*$ & $*$ & $*$ \\
\hline$T_{12}$ & $*$ & $*$ & 1 & $\times$ & $\times$ & $\times$ & $\times$ & $*$ & $*$ & $*$ & $*$ \\
\hline$T_{13}$ & $*$ & 1 & $*$ & $\times$ & $*$ & $*$ & $\times$ & $*$ & $*$ & $\times$ & $*$ \\
\hline$T_{14}$ & $\times$ & $*$ & $\times$ & $*$ & $\times$ & $\times$ & $*$ & $\times$ & $*$ & 1 & $*$ \\
\hline$T_{15}$ & $*$ & $*$ & $*$ & $\times$ & 1 & $*$ & $\times$ & $*$ & $*$ & $\times$ & $*$ \\
\hline
\end{tabular}

TABLE 9. FeTAR instance results.

\begin{tabular}{|c|c|c|c|c|c|c|c|c|c|c|c|c|c|c|}
\hline & $S_{3}$ & $S_{4}$ & $S_{5}$ & $S_{7}$ & $S_{8}$ & $S_{9}$ & $S_{14}$ & $S_{15}$ & $S_{16}$ & $S_{17}$ & $S_{18}$ & $W$ & $D_{w}$ & $C_{w}$ \\
\hline$T_{2}$ & $*$ & $*$ & $\times$ & $*$ & $*$ & $*$ & 1 & $*$ & $\times$ & $*$ & $*$ & 1 & 539.3 & 39 \\
\hline$T_{3}$ & $*$ & $*$ & $*$ & $*$ & $*$ & $*$ & $*$ & $*$ & $*$ & $\times$ & 1 & 2 & 539.3 & 41 \\
\hline$T_{5}$ & 1 & $*$ & $*$ & $*$ & $*$ & $*$ & $*$ & $\times$ & $*$ & $*$ & $*$ & 3 & 539.3 & 47 \\
\hline$T_{6}$ & $*$ & $*$ & $*$ & 1 & $*$ & $*$ & $\times$ & $*$ & $*$ & $*$ & $*$ & 4 & 539.3 & 22 \\
\hline$T_{7}$ & $*$ & $*$ & $\times$ & $\times$ & $*$ & 1 & $*$ & $*$ & $\times$ & $\times$ & $*$ & & & \\
\hline$T_{8}$ & $*$ & $*$ & $\times$ & $*$ & $*$ & $*$ & $*$ & $*$ & $*$ & $*$ & 1 & & & \\
\hline$T_{9}$ & $\times$ & $*$ & $\times$ & $\times$ & 1 & $*$ & $\times$ & $*$ & $\times$ & $*$ & $\times$ & & & \\
\hline$T_{10}$ & $\times$ & $*$ & $*$ & $\times$ & $\times$ & $*$ & $*$ & $\times$ & 1 & $*$ & $\times$ & & & \\
\hline$T_{11}$ & $*$ & $*$ & $*$ & $*$ & $*$ & $*$ & $*$ & 1 & $*$ & $*$ & $*$ & & & \\
\hline$T_{12}$ & $*$ & $*$ & 1 & $\times$ & $\times$ & $\times$ & $\times$ & $*$ & $*$ & $*$ & $*$ & & & \\
\hline$T_{13}$ & $*$ & 1 & $*$ & $\times$ & $*$ & $*$ & $\times$ & $*$ & $*$ & $\times$ & $*$ & & & \\
\hline$T_{14}$ & $\times$ & $*$ & $\times$ & $*$ & $\times$ & $\times$ & $*$ & $\times$ & $*$ & 1 & $*$ & & & \\
\hline$T_{15}$ & $*$ & $*$ & $*$ & $\times$ & 1 & $*$ & $\times$ & $*$ & $*$ & $\times$ & $*$ & & & \\
\hline
\end{tabular}

\section{COMPARison}

Many researchers have longer discussed the benefits of the MA paradigm and conclude that it might be efficient in some cases [28,29]. In a recent Work [30], we compared our approach with the use of the classical CS paradigm. Results are very significant. Through our system, we deduce final Workplans of CA agents according the generated FeTAR instances. Therefore, in order to response to all users' requests, the generated solution (Tab. 8) required $539.3 \mathrm{~ms}$ in the MA paradigm (Tab. 9) and $4076.67 \mathrm{~ms}$ in the CS one (Fig. 7). Besides, when we 


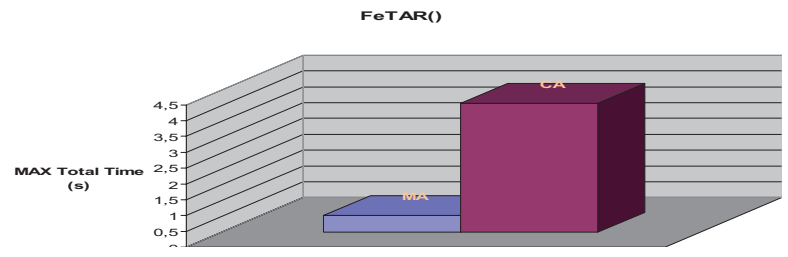

FiguRE 7. FeTAR instance result.

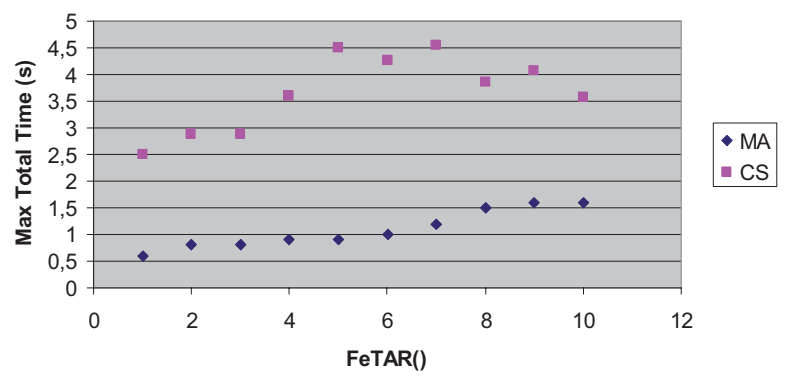

Figure 8. Variation of FeTAR instance.

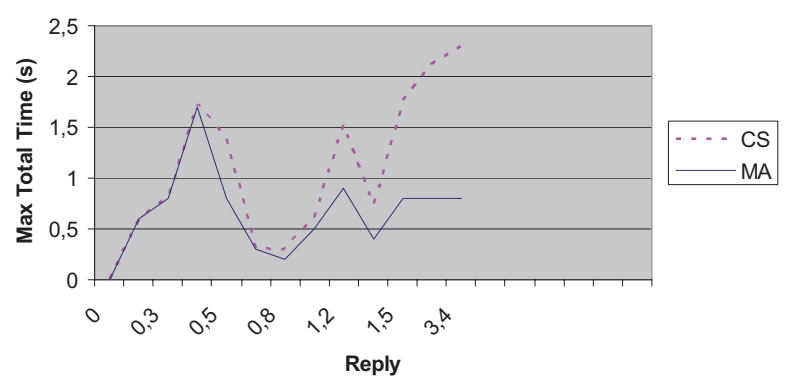

FIGURE 9. Variation of response data.

randomly generate several FeTAR instances for the same example, we observe the benefit of the use of our optimization approach, using MA paradigm instead of the CS one through our system (Fig. 8). In addition, when the quantity of response data varies, we observe that the use of MA paradigm is better (Fig. 9). The graphic is not regular because we solve the problem in a general case so we used random latencies.

\section{THE JADE PLATFORM}

We are developing our system, with JADE platform (Java Agent Development platform) [23]. JADE is a middleware which permits a flexible implementation of multi-agents systems; it offers an efficient transport of ACL (Agent Communication Language) messages for agents communication which complies with FIPA 
specifications [25]. A yellow pages services directory is available, allowing agents to advertise their services; visiting agents can then consult yellow pages looking for agents which provide the services they desire. JADE is written in java language [24], supports mobility, evolves rapidly and until there, it is the only existent multi-agent platform which tolerates web services integration [26].

\section{Conclusion}

In this paper, we proposed a migration strategy of MAs through a MN, optimizing the data flow management, in order to satisfy, in a better manner, customers' requests. The adopted approach decreases considerably computing time because Workplans are just deduced; they are computed when network traffic varies considerably. We aim to improve our approach according to eventual similarities, resulting from simultaneous requests sets overlap. For this purpose, we aim to detail relationships between different agents of our system, implementing their different possible kinds of cooperation.

\section{REFERENCES}

[1] H. Zgaya, K. Ghédira and S. Hammadi, Proposition d'un système d'information multimodal à base d'agents mobiles destiné aux clients des réseaux de transport, in Proc. of the International Workshop of Méthodologies et Heuristiques pour l'Optimisation des Systèmes Industriels (MHOSI'05), Hammamet, Tunisia 24-26 April (2005).

[2] S. Rouvrais, Utilisation d'agents mobiles pour la construction de services distribués. Ph.D. Thesis, University of Rennes, France (2002).

[3] W. Theilmann and K. Rothermel, Efficient Dissemination of Mobile Agents, in Proc. of the 19th IEEE International Conference on Distributed Computing Systems Workshops (ICDCSW'99), edited by W. Sun, S. Chanson, D. Tygar and P. Dasgupta, Austin, TX, USA, 31 May-5 Juin (1999) 9-14.

[4] A. Fugetta, G.P. Picco and G. Vigna, Understanding code mobility, IEEE Trans. Software Engineering 24(5) (1998) 342-361.

[5] A. Carzaniga, G.P. Picco and G. Vigna, Designing distributed applications with mobile code paradigms, in Proc. of the 19th International Conference on Software Engineering (ICSE'97), Boston, Massachusetts, USA 17-23 May (1997). ACM Press (1997) 22-32.

[6] D. Rus, R. Gray and D. Kotz, Autonomous and adaptive agents that gather information, in Proc. of the Thirteen National Conference on Artificial Intelligence Workshop on Intelligent Adaptive Agents (AAAI'96), Portland, Oregon, USA, 4-8 August (1996) 107-116.

[7] J. Yang, V. Honavar, L. Miller and J. Wong, Intelligent Mobile Agents for information retrieval and knowledge discovery from distributed data and knowledge sources, in Proc. of the IEEE Information Technology Conference, Syracuse, NY USA, 1-3 September (1998) $99-102$.

[8] S. Green, L. Hurst, B. Nangle, P. Cunningham, F. Somers and R. Evans, Software agents: A review. Technical report, TCS-CS-1997-06, Trinity College Dublin, Ireland (1997).

[9] D. Kotz and R.S. Gray, Mobile Agents and the Future of the Internet, Department of Computer Science, Thayer School of Engineering, Dartmouth College, Hanover, New Hampshire 03755, ACM SIGOPS Operating Systems Review 33(3) (1999) 7-13.

[10] G. Bernard, Technologie du code mobile: état de l'art et perspectives, in Proc. Of the Colloque Francophone sur l'Ingénierie des Protocoles (CFIP'99), Nancy, France April 2629 (1999). 
[11] K. Moizumi, Mobile Agent Planning Problems. Ph.D. Thesis, Thayer School of Engineering, Dartmouth College, Hanover, NH 03755 USA (1998).

[12] W. Caripe, G. Cybenko, K. Moizumi and R. Gray, Dartmouth Coll., Hanover, NH; Network awareness and mobile agent systems. IEEE Comm. Magazine, 36-7 (1998) 44-49.

[13] K. Moizumi and G. Cybenko, The traveling agent problem. Mathematics of Control, Signals and Systems (1998).

[14] H. Zgaya, S.Hammadi and K. Ghédira, Workplan Mobile Agent for the Transport Network Application, in Proc. of 17th IMACS World Congress Scientific Computation, Applied Mathematics and Simulation (IMACS'2005), Paris 11-15 July, (2005).

[15] E. Horowitz and S.Sahni, Fundamentals of Computer Algorithms. Computer Science Press (1989).

[16] J.W. Baek, J.H. Yeo, G.T. Kim and H.Y. Yeom, Cost effective mobile agent planning for distributed information retrieval, in Proc. of 21 st International Conference on Distributed Computing Systems (ICDCS'01), Phoenix, Arizona USA, 16-19 April (2001) 65-72.

[17] M. Garey and D. Johnson, Computers and Intractability: A Guide to the theory of NPCompleteness, W. H. Freeman and Co., USA (1979).

[18] Z. Michalewicz, Genetic Algorithms + Data Structures $=$ Evolution programs. Springer Verlag (1992).

[19] H. Zgaya, S. Hammadi and K. Ghédira, Evolutionary method to optimize Workplan mobile agent for the transport network application, in Proc. of International Conference on Systems, Man and Cybernetics, (SMC'2005) 10-12 October Hawaii, USA, 2(2005) 1174-1179.

[20] L. Davics, Handbook of genetic algorithm. New York: Van Nostrand Reinhold (1991).

[21] M. Purvis, S. Crenefield, R. Ward, M. Nowostawski, D. Carter and G. Bush, A multiagent system for the integration of distributed environmental information, Environmental Modelling $\mathcal{E}$ Software,Information Science Department, University of Otago, Dunedin, New Zealand, 18(6) (2003) 565-572.

[22] E. Zitzler and L. Thiele, Multiobjective Optimization Using Evolutionary Algorithms: A Comparative Case Study, in Lect. Notes Comput. Sci. 1498 UK (1998) 292-301.

[23] Java Agent DEvelopment framework. http://jade.titlab.com/doc

[24] www. java.sun.com

[25] www.fipa.org

[26] D. Greenwood, JADE Web Service Integration Gateway (WSIG), WHITESTEIN Technologies. Jade Tutorial, in Fourth International Joint Conference on Autonomous Agents and Multi Agent Systems (AAMAS 2005), Utrecht University, the Netherlands, 25-29 July (2005).

[27] K. Deb, S. Agrawal, A. Pratap and T. Meyarivan, A fast and elitist multi-objective genetic algorithm: NSGA-II, IEEE Transactions on Evolutionary Computation, 6(2) (2002) 182 197.

[28] G.P. Picco and M. Baldi, Evaluating the Tradeoffs of Mobile Code Design Paradigms in Network Management Applications, in Proc. of 20th IEEE International Conference on Software Engineering (ICSE'97), edited by R. Kemmerer and K. Futatsugi, Kyoto, Japan, April (1998) 146-155.

[29] M. Ketel, N.S. Dogan and A. Homaifar, Distributed Sensor Networks based on Mobile Agents Paradigms, Dept. of Computer Science, North Carolina A\&T State University, NC 27411, in Proc. of the Thirty-Seventh Southeastern Symposium on System Theory (SSST'05), Greensboro, USA, 20-22 March (2005) 411-414.

[30] H. Zgaya and S. Hammadi, Assignment and Integration of Distributed Transport Services in Agent-Based Architecture, IEEE/WIC/ACM International Conference on Intelligent Agent Technology (IAT 2006 Main Conference Proceedings) (IAT'06), Hong Kong, China, 18-22 December (2006) 96-102. 